\title{
Inclusive Diffraction at HERA
}

On behalf of H1 and ZEUS collaborations

\author{
Laurent Schoeffel \\ CEA Saclay, Irfu/SPP
}

Paris, 21rst May 2013 


\section{Diffraction on nuclear waves}

\section{ELASTIC AND INELASTIC SCATTERING OF $1.37 \mathrm{GeV} \propto$-PARTICLES FROM ${ }^{40,42,44,48} \mathrm{Ca}$}

G. D. ALKHAZOV ${ }^{\star}$, T. BAUER ${ }^{\dagger}$, R. BERTINI ${ }^{*}$, L. BIMBOT ${ }^{\ddagger}$, O. BING ${ }^{*+}$, A. BOUDARD,

G. BRUGE, H. CATZ, A. CHAUMEAUX, P. COUVERT, J. M. FONTAINE ${ }^{*}$, F. HIBOU ${ }^{*}$,

G. J. IGO $\sharp *$, J. C. LUGOL and M. MATOBA*

Service de Physique Nucléaire à Moyenne Energie

CEN Saclay, BP 2, 91190 Gif-sur-Yvette, France

Received 6 October 1976

(Revised 9 December 1976)

$\vartheta\left(\right.$ or ||$\left.^{1 / 2}\right)$ dependence presents the standard diffractive pattern (optics)

Amplitude $(q, k) \sim i k / 2 \pi \int d b e^{i b q} D(b, k)$

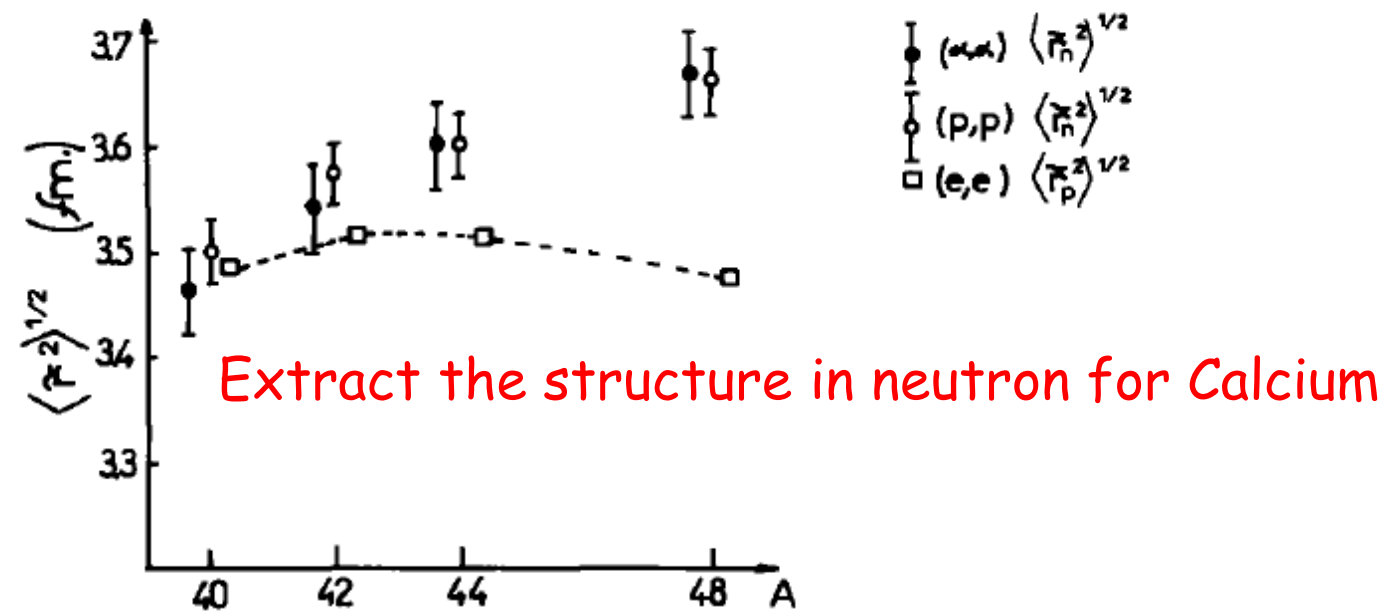

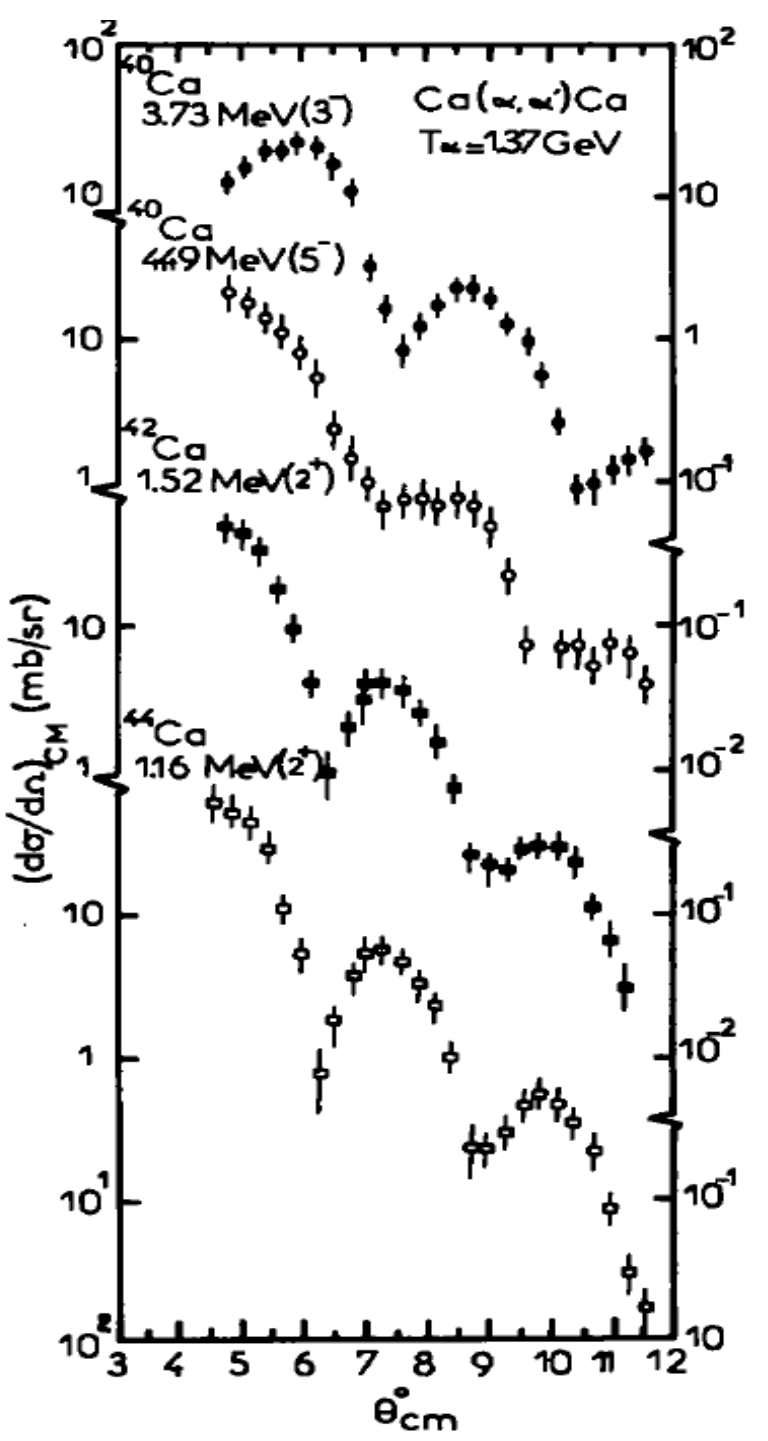

Fig. 3. Differential cross sections of inelastic scattering of $1.37 \mathrm{GeV} \alpha$-particles from the $3-$ and $5_{1}^{-}$states in ${ }^{40} \mathrm{Ca}$ and the $2_{1}^{+}$states in ${ }^{42} \mathrm{Ca}$ and ${ }^{44} \mathrm{Ca}$. 


\section{Subnuclear waves}

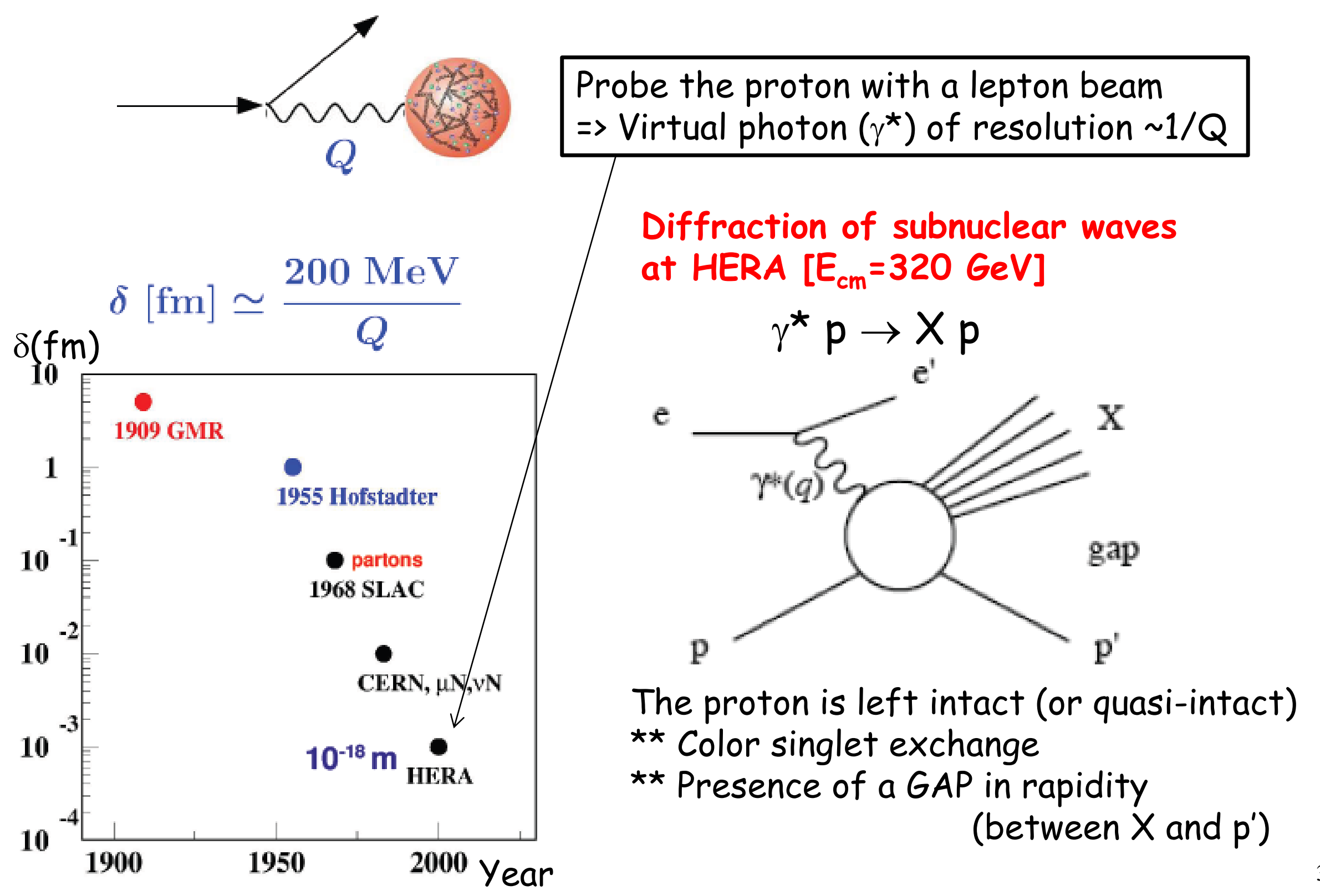




\section{HERA-DESY: 1992-2007}

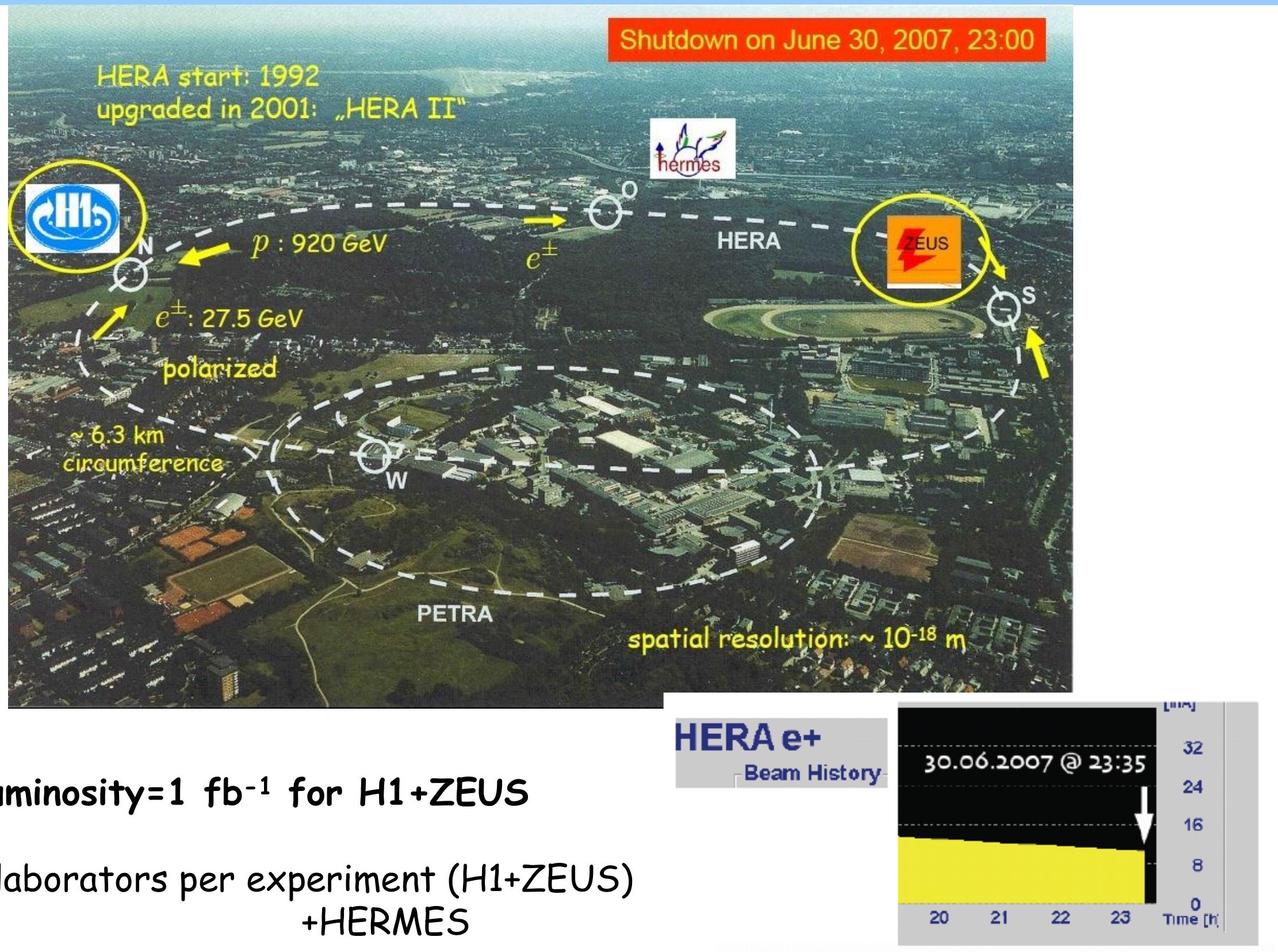

Total luminosity $=1 \mathrm{fb}^{-1}$ for $\mathrm{H} 1+$ ZEUS

350 collaborators per experiment (H1+ZEUS) +HERMES 


\section{Diffractive events are observed}

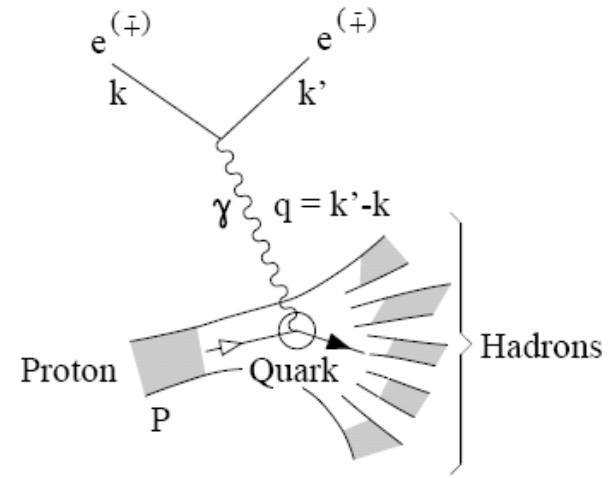

Deep Inelastic Scattering (DIS) $\Rightarrow F_{2}$

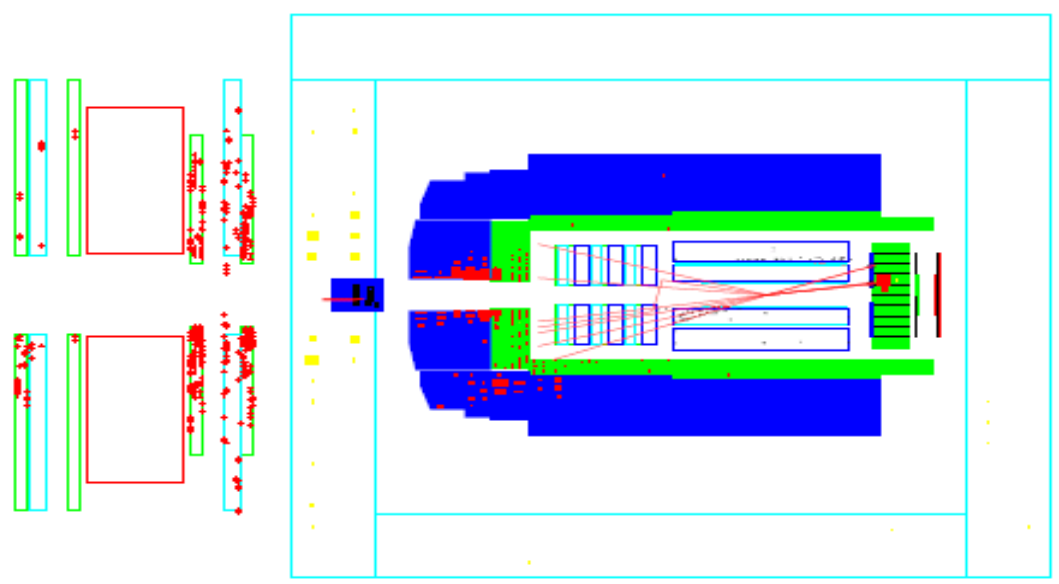

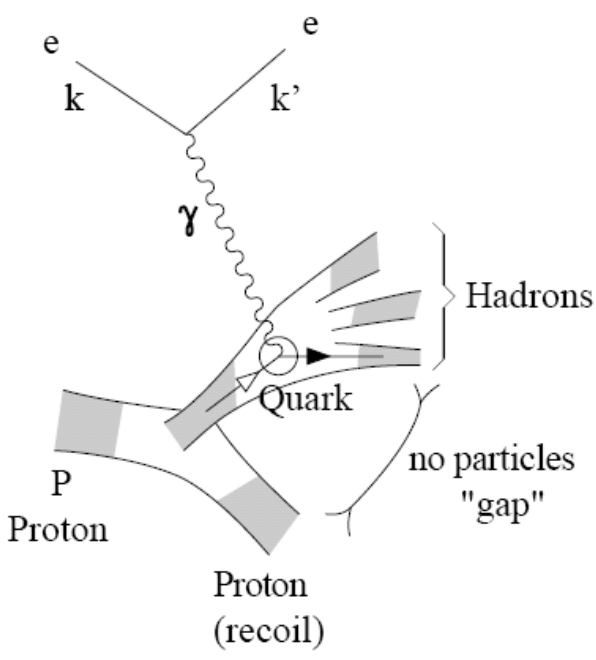

Diffractive Deep Inelastic Scattering (DDIS) $\Rightarrow F_{2}{ }^{D}$

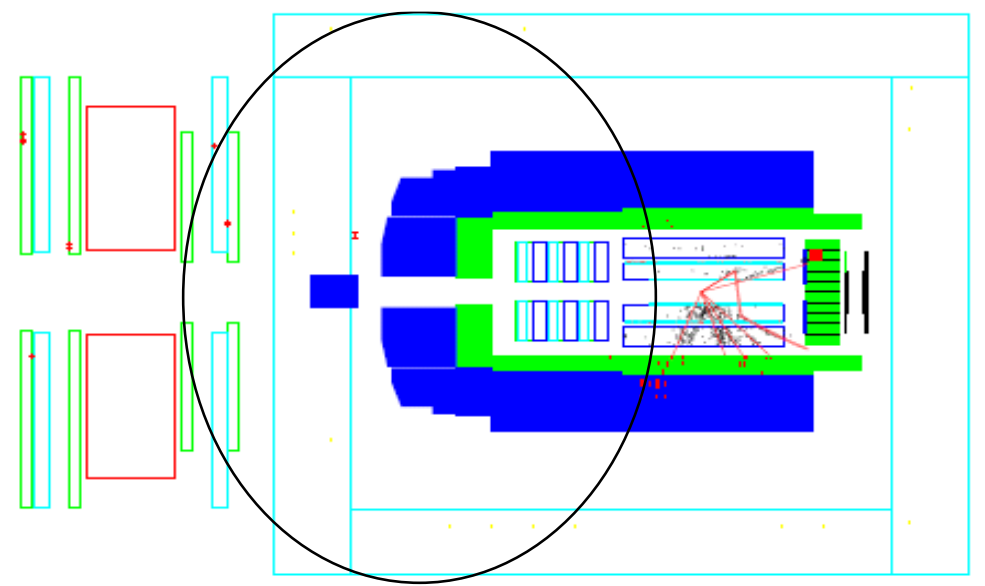

This is the GAP with no particle 


\section{Experimental selection methods}

Scattered proton in Leading Proton Spectrometers (LPS)

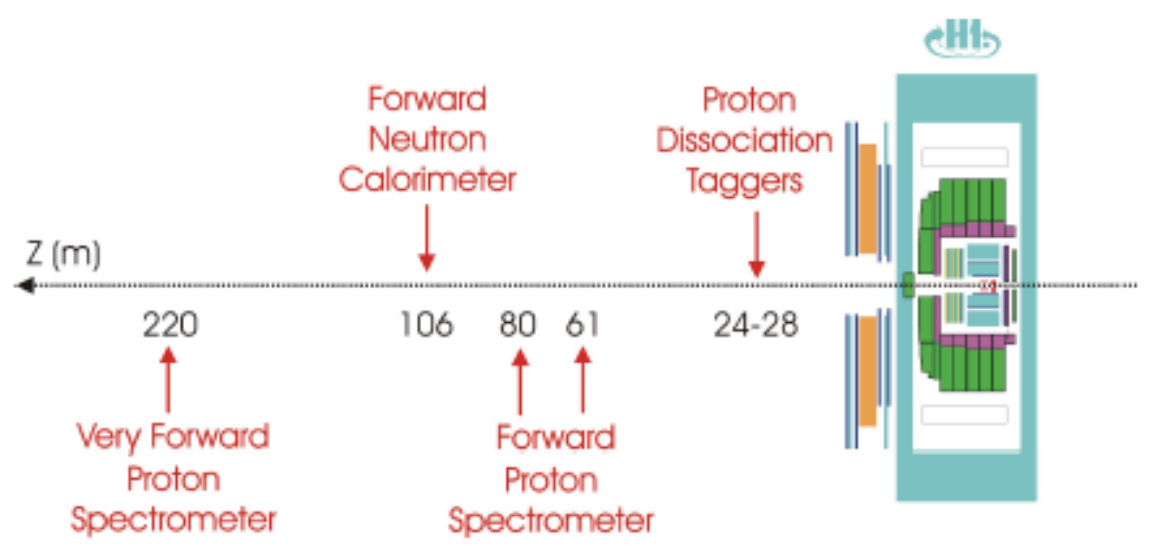

Limited by statistics and p-tagging systematics
'Large Rapidity Gap' (LRG) adjacent to outgoing (untagged) proton

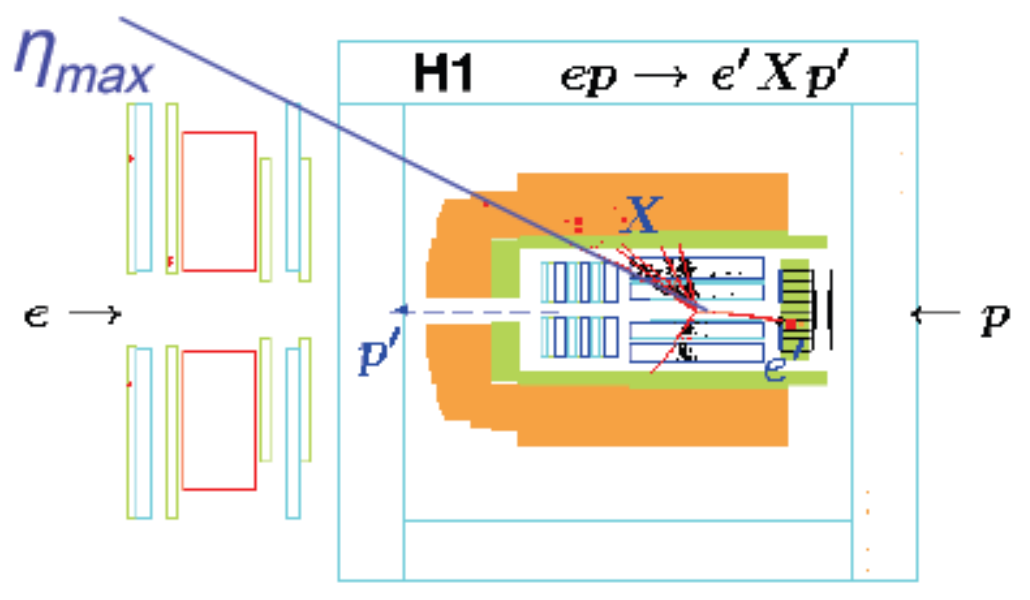

Limited by p-diss systematics 


\section{Diff events are produced with a quite large rate}

- Data : 1241193

MC : 1228063.125

VM: 4290.088

Lower $\eta_{\text {MAX }}$ means that the GAP with no particle is large NC: 119402.484 IPcha: 152679.438 ...illustration on all HERAII data (Lumi $=330 \mathrm{pb}^{-1}$ )
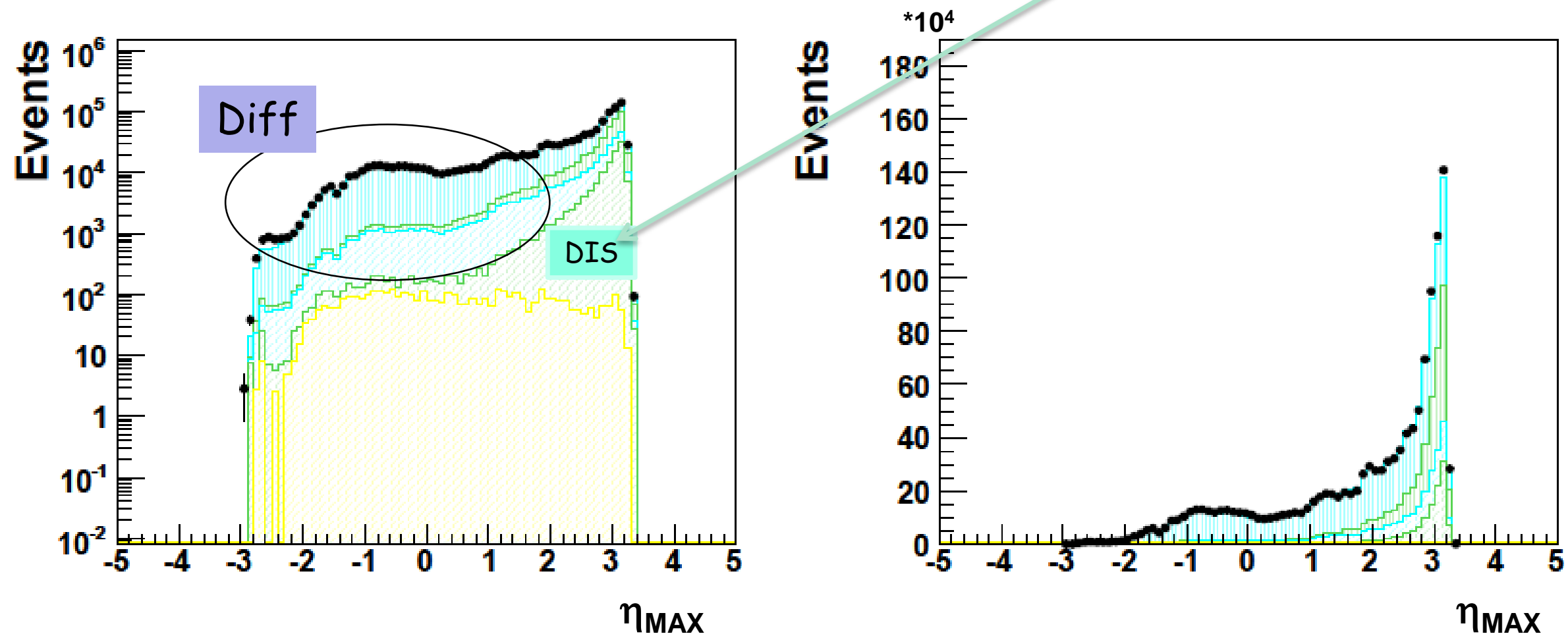


\section{Why DIFF rate is large @ HERA (low $x)$ ?}

...certain (Fock) states of the virtual photon $\left|\psi_{k}\right\rangle$ do not feel the the strong interaction, while others are strongly affected...

$\Rightarrow$ Large fluctuations in the absorbption coefficients of these states...

This is (obviously) linked to the dominance of the gluon density at small $x$.

It finds a natural extension in the dipole approach:

$T(b) \sim \alpha_{S} r^{2} \times G\left(x, 1 / r^{2}\right) /\left(\pi R^{2}\right) * \exp \left(-b^{2} / b_{0}{ }^{2}\right)$
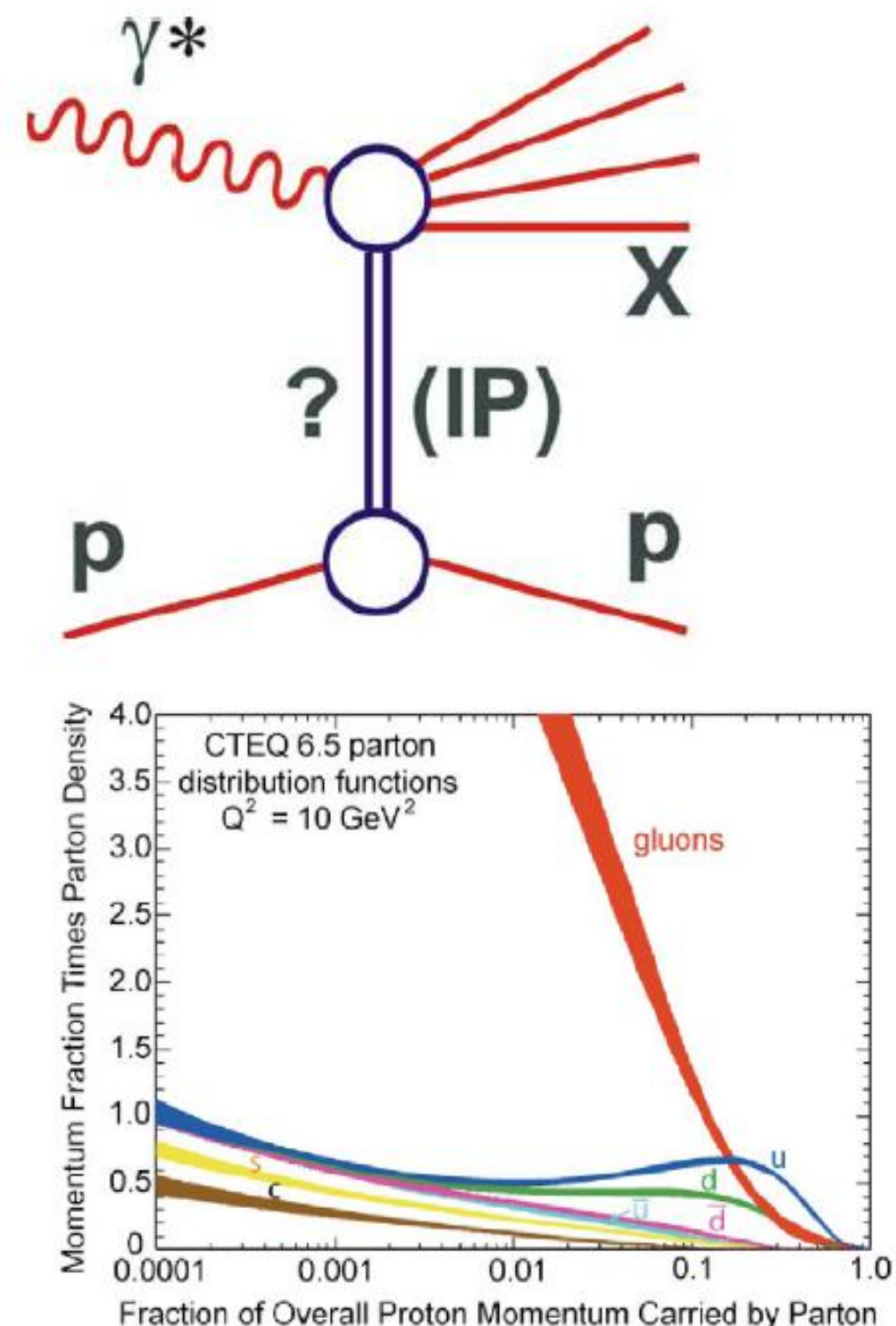


\section{Kinematics and notations}

Standard DIS variables ...

$x=$ momentum fraction $q / p$

$Q^{2}=\mid \gamma^{\star} 4$-momentum squared $\mid$

Additional variables for diffraction ...

$t=$ squared 4-momentum transfer at proton vertex

$x_{I P}=$ fractional momentum loss of proton (momentum fraction IP/p)

$\beta=x / x_{\text {IP }}$

(momentum fraction $q / I P$ )
Most generally ep $\rightarrow$ eXY ...

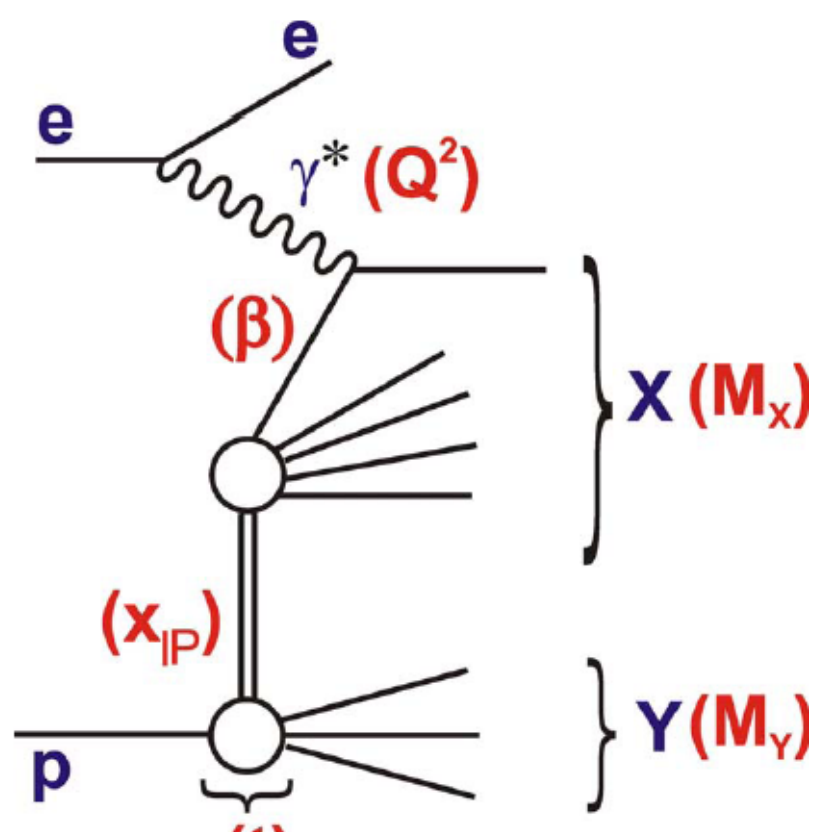

(t)

In most cases here, $y=p$, (small admixture of low mass excitations) 


\section{Diffractive cross sections (definition)}

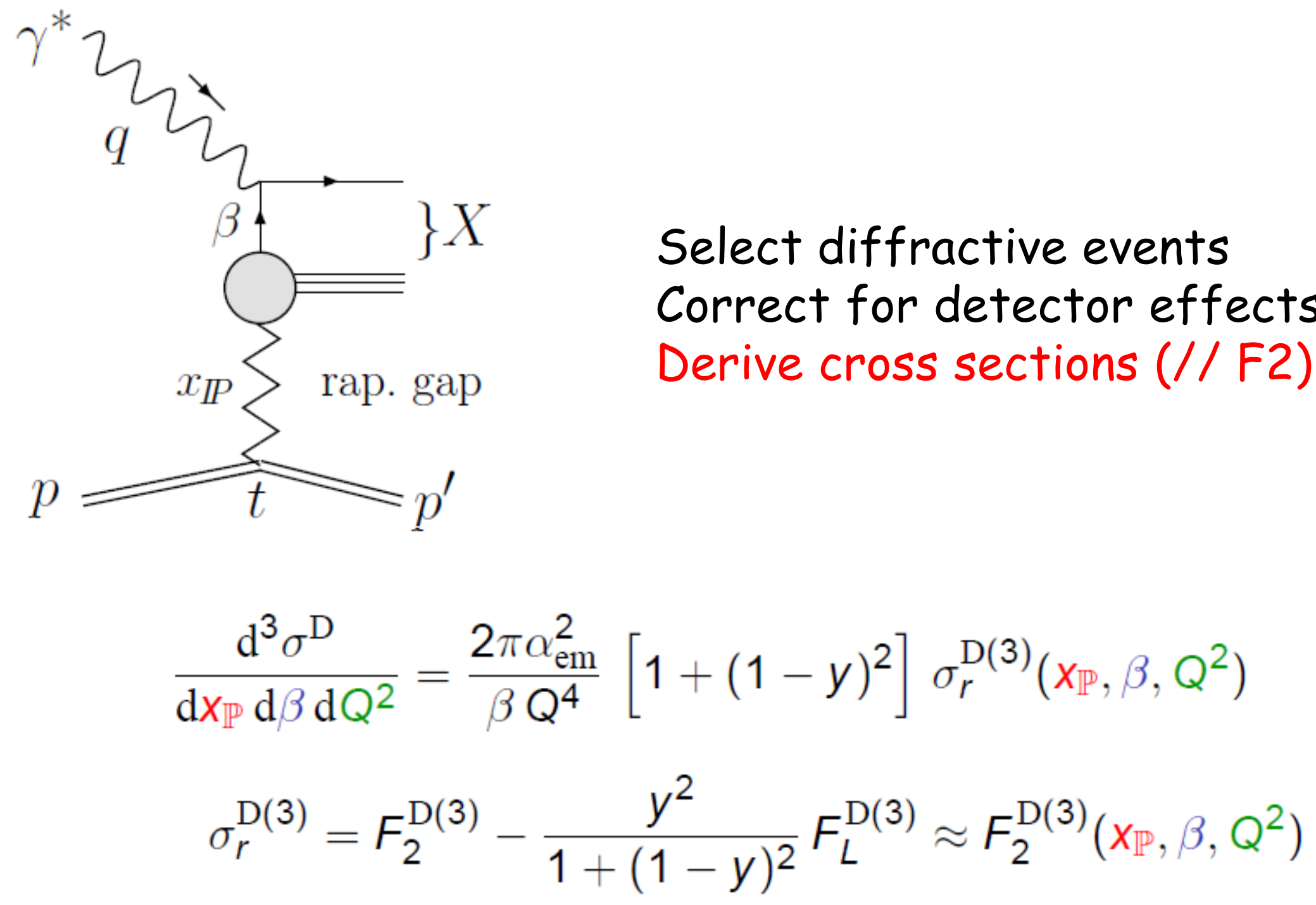




\section{H1 LRG samples}

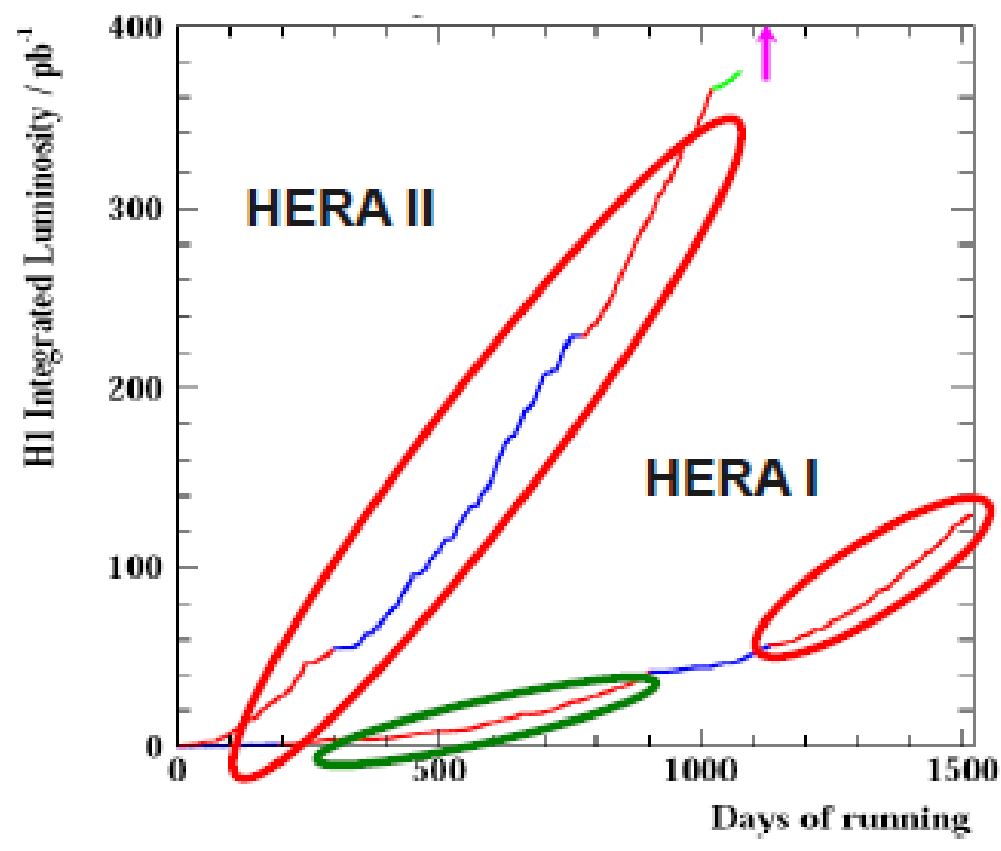

\begin{tabular}{|l|c|c|c|}
\hline \multicolumn{1}{|c|}{ Data Set } & $\begin{array}{c}Q^{2} \text { range } \\
\left(\mathrm{GeV}^{2}\right)\end{array}$ & $\begin{array}{c}\text { Proton Energy } \\
E_{p}(\mathrm{GeV})\end{array}$ & $\begin{array}{c}\text { Luminosity } \\
\left(\mathrm{pb}^{-1}\right)\end{array}$ \\
\hline \hline \multicolumn{4}{|c|}{ New data samples } \\
\hline 1999 MB & $3<Q^{2}<25$ & 920 & 3.5 \\
$1999-2000$ & $10<Q^{2}<105$ & 920 & 34.3 \\
$2004-2007$ & $10<Q^{2}<105$ & 920 & 336.6 \\
\hline \multicolumn{4}{|c|}{ Prev10usy published data samples } \\
\hline $1997 \mathrm{MB}$ & $3<Q^{2}<13.5$ & 820 & 2.0 \\
1997 & $13.5<Q^{2}<105$ & 820 & 10.6 \\
$1999-2000$ & $133<Q^{2}<1600$ & 920 & 61.6 \\
\hline
\end{tabular}

[H1 Coll. EPJC28 (2006) 715]

- All H1 data samples now analysed

$\rightarrow$ Increase in statistics of 3 to 30

- All combined into one single H1 LRG cross section set

\ Total kinematic range:

$$
\begin{aligned}
& 3.5<\mathrm{Q}^{2}<1600 \mathrm{GeV}^{2} \\
& 0.0017<\beta<0.8 \\
& 0.0003<\mathrm{x}_{\mathrm{IP}}<0.03
\end{aligned}
$$




\section{Combination of H1 LRG data}

- Combine reduced cross sections from each data period

- Iterative $x^{2}$ minimisation used

- Full error correlations considered

$\rightarrow 597$ data points averaged to 277 measurements

$\rightarrow x^{2} /$ ndof $=371 / 320$

- Pulls of individual points to combined points

$\rightarrow$ No large tension between data sets observed

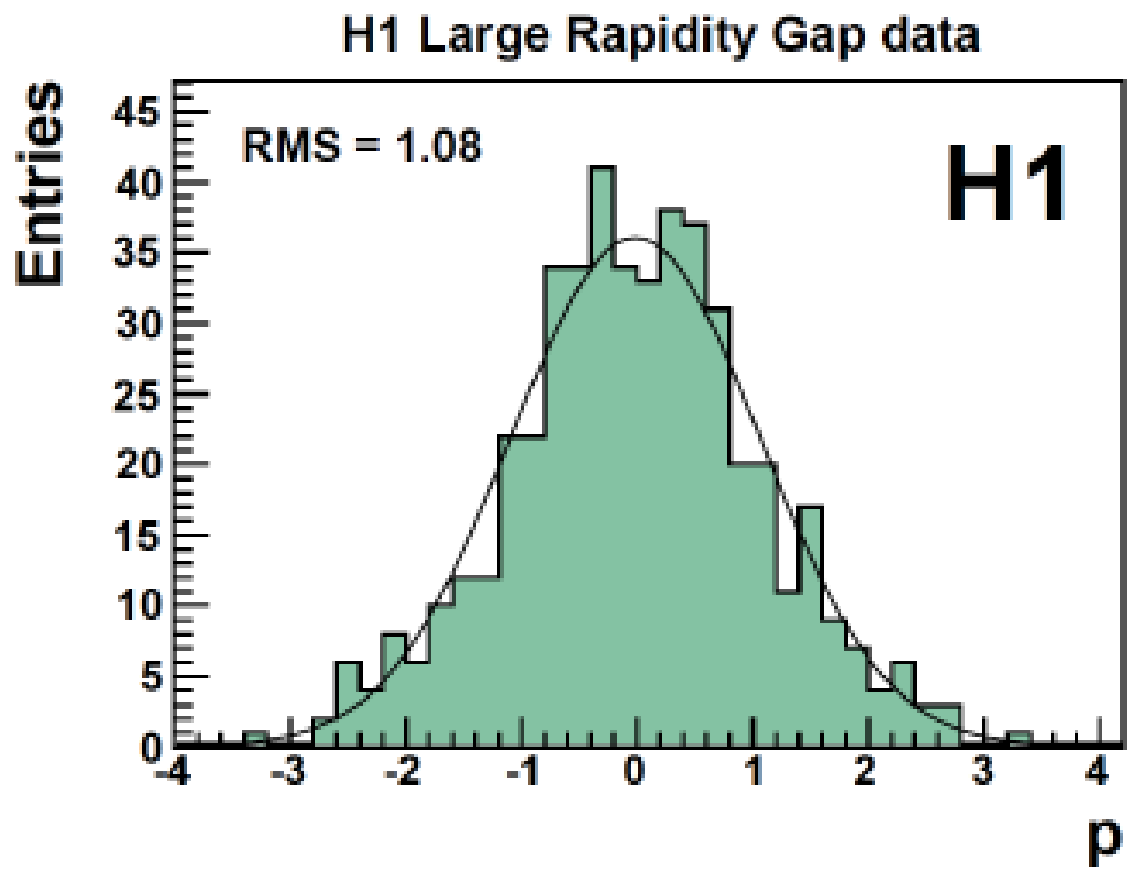




\section{Combined H1 LRG cross section $\left(F 2^{D}\right)\left[Q^{2}\right]$}

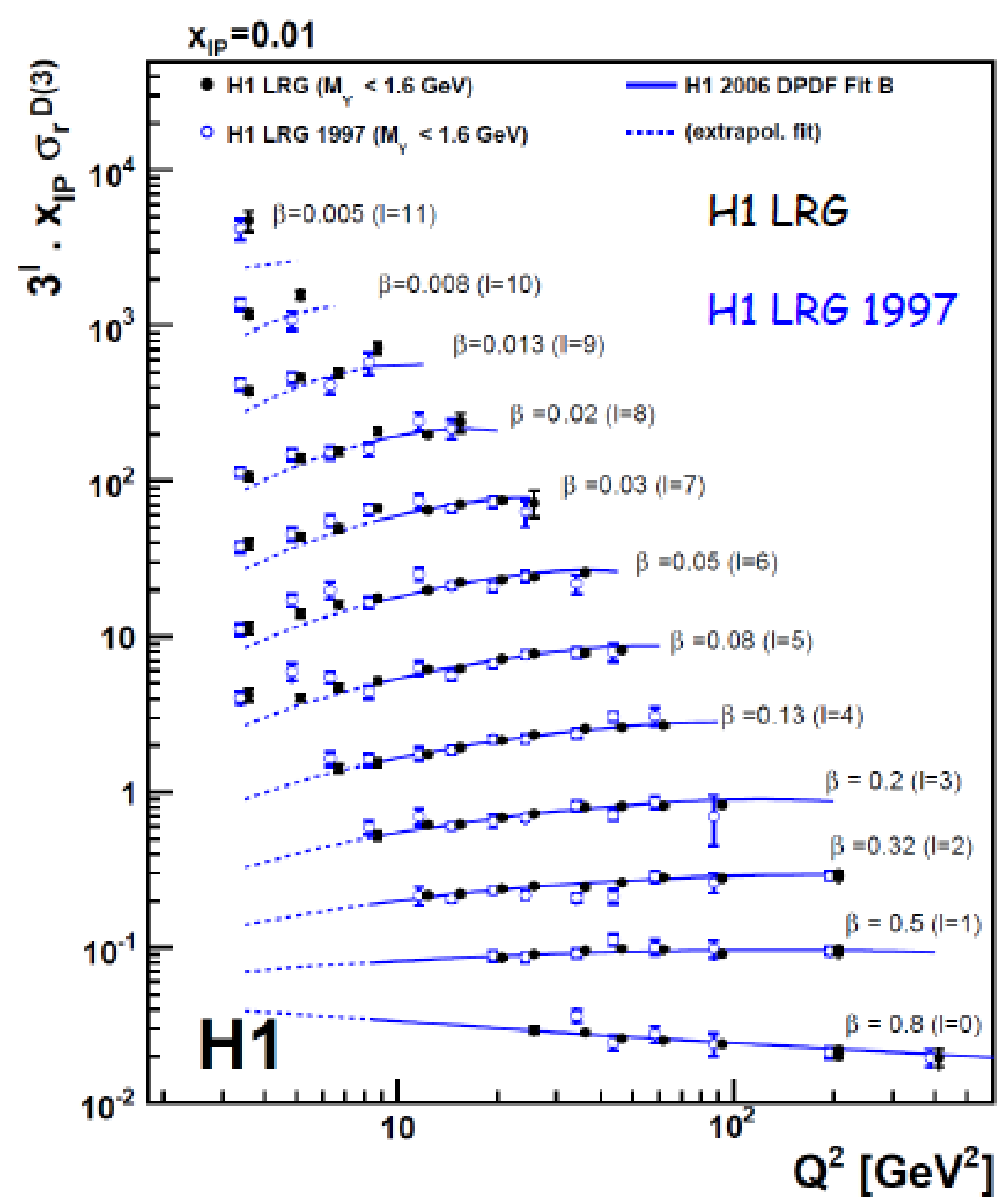

- Example of $Q^{2}$ dependence for $X_{\mathbb{P}}=0.01$

$\rightarrow$ Large reduction of statistical errors

$\rightarrow$ Typical precision for $\mathrm{Q}^{2}>12 \mathrm{GeV}^{2}$ :
$1 \%$ (stat.)
$5 \%$ (sys.)
$4 \%$ (norm.) 


\section{$F 2$ versus $F_{2}{ }^{D}$}

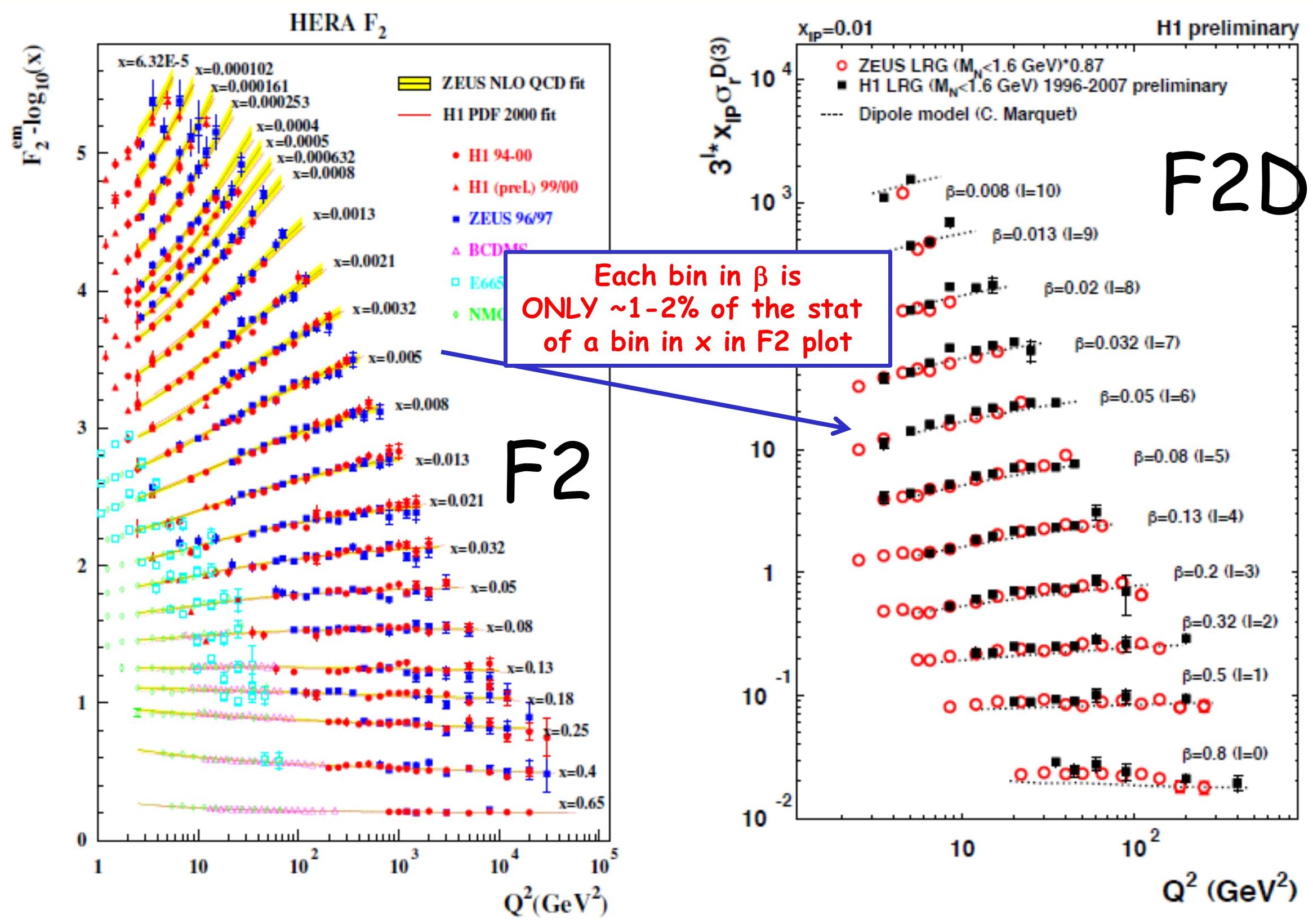

14 


\section{Combined $H 1 L R G$ cross section $\left(F 2^{D}\right)[\beta]$}

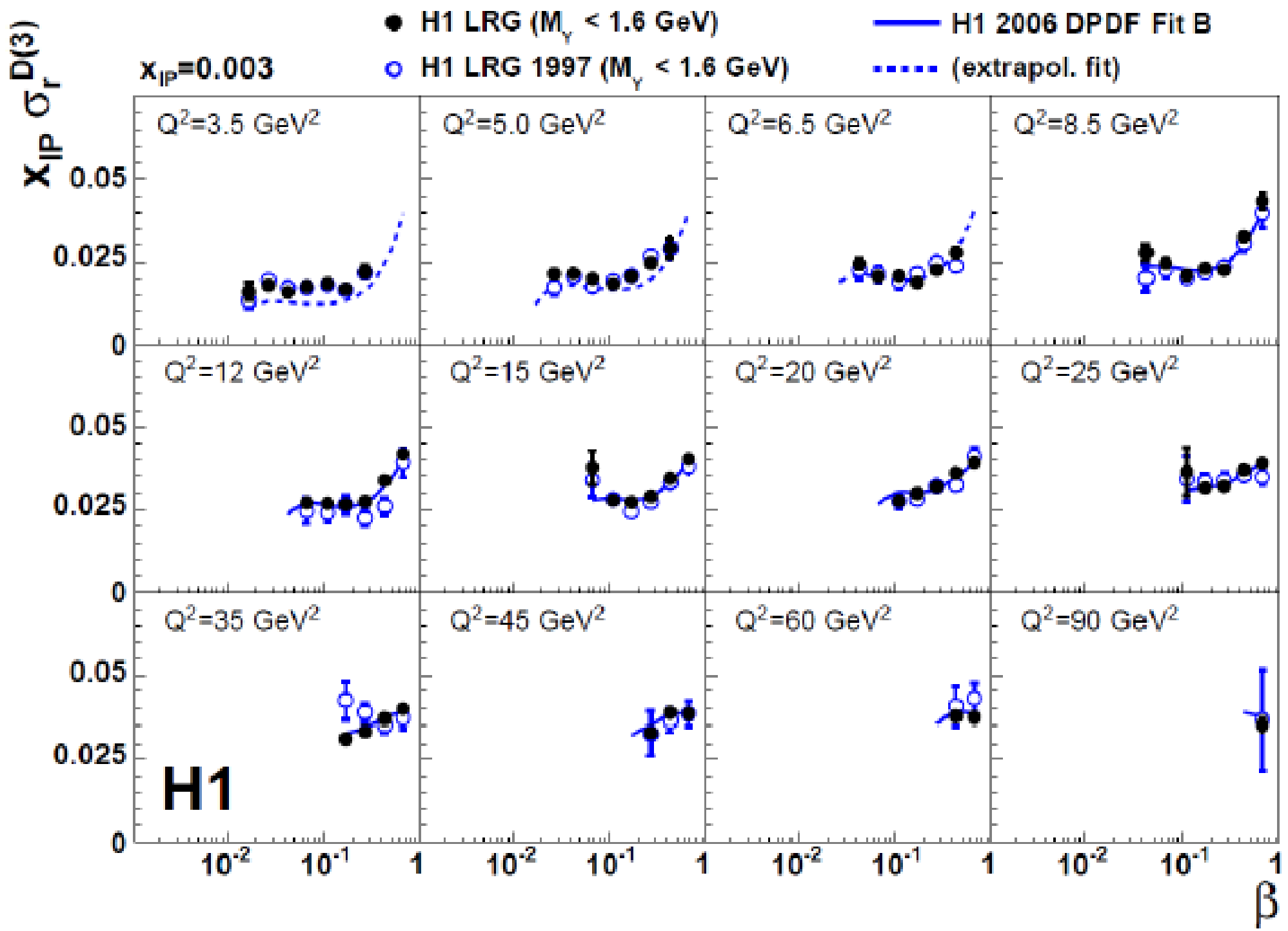

H1 LRG

H1 LRG 1997 


\section{$L R G$ versus $p$-tagged F2D}

- Compare H1 LRG and FPS cross sections

$\rightarrow$ Ratio LRG / FPS :

$\frac{\sigma\left(M_{Y}<1.6 \mathrm{GeV}\right)}{\sigma(Y=p)}=$
$1.203 \pm 0.019$ (exp.) \pm 0.087 (norm.)
$(1.6 \%) \quad(7.2 \%)$

$\rightarrow$ Experimental control of the amount of proton dissociation in LRG data

$\rightarrow$ No $\beta$ or $Q^{2}$ dependent differences observed

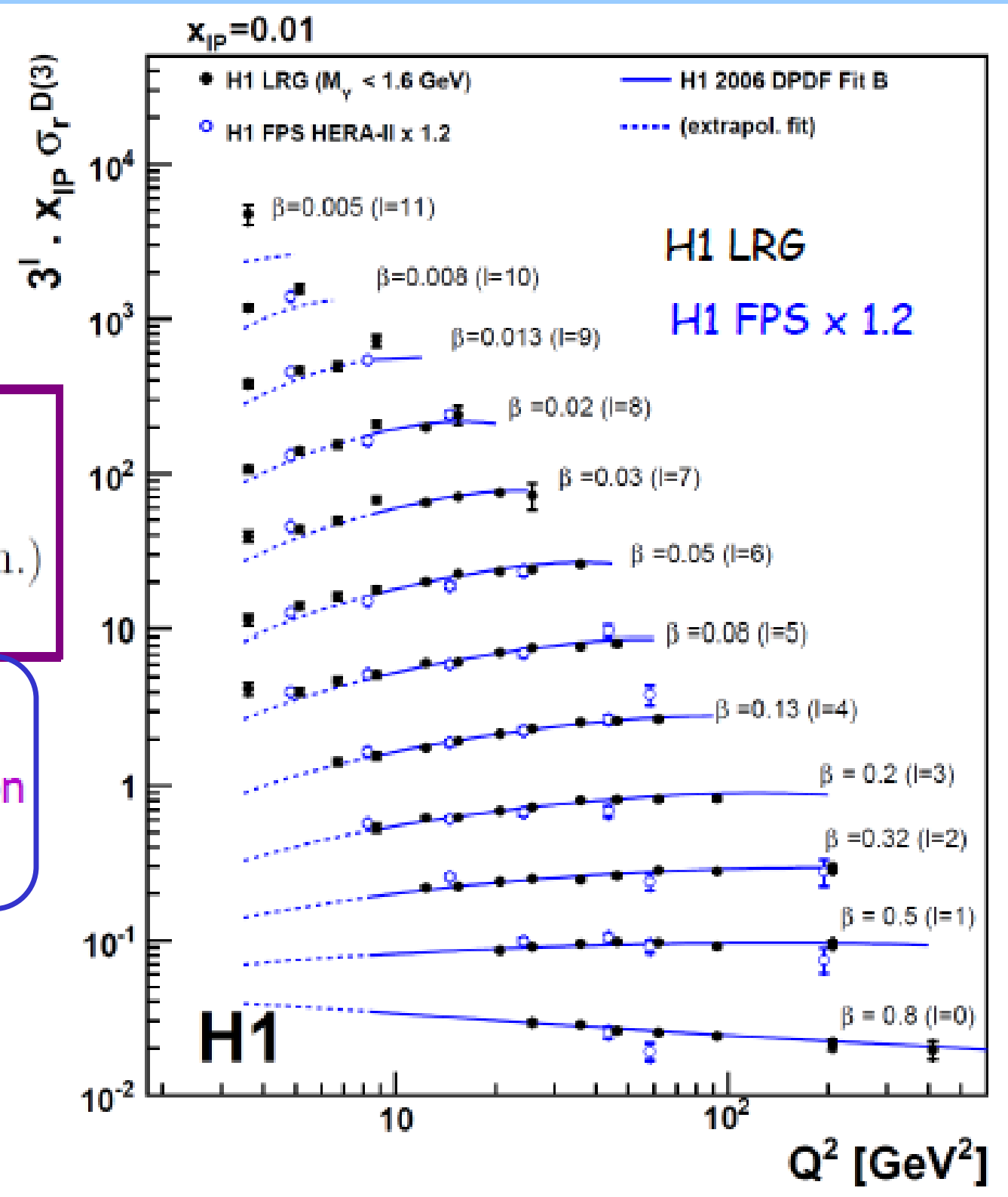




\section{Experimental summary for $\mathrm{H}_{1} \mathrm{~F}_{2}{ }^{\mathrm{D}}$}

\section{H1 PRELIMINARY}

It took $>15$ years of analysis to reach this:

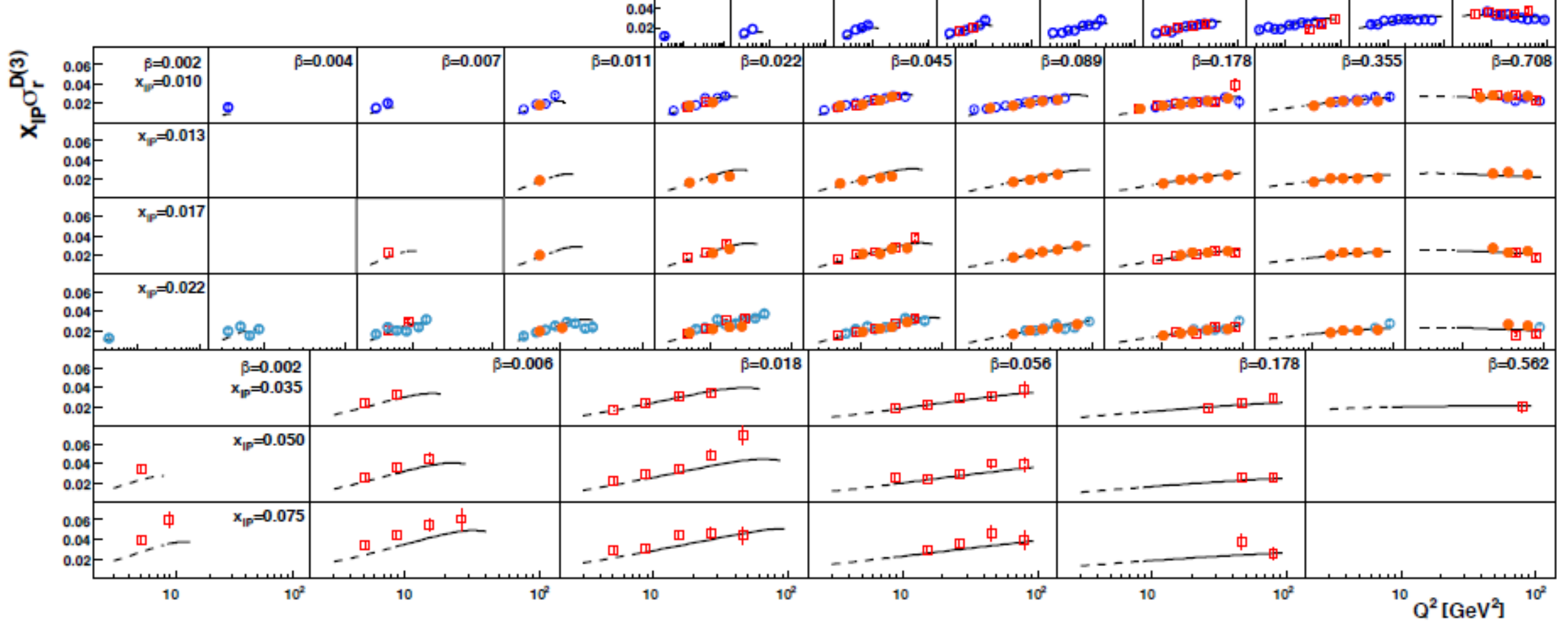




\section{$\mathrm{H} 1$ and ZEUS data on $\mathrm{F}^{\mathrm{D}}$}

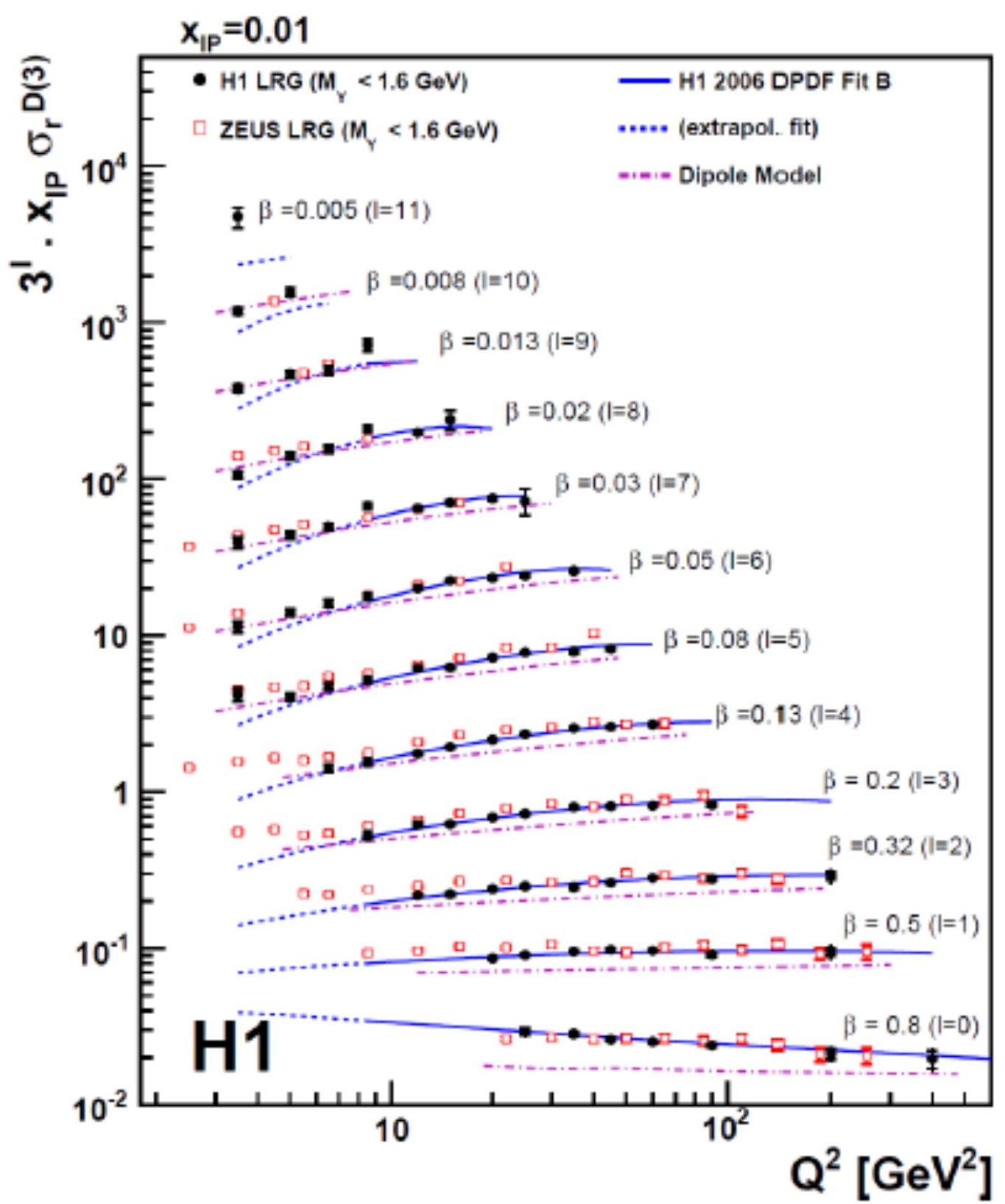

H1 LRG $\left(M_{\mathrm{y}}<1.6 \mathrm{GeV}^{2}\right)$

ZEUS LRG $\left(M_{\mathrm{y}}<1.6 \mathrm{GeV}^{2}\right)$

- ZEUS data rescaled to $M_{\gamma}<1.6 \mathrm{GeV}^{2}$

[ZEUS Coll. NPB816 (2009) 1]

- General overall agreement

- Overall 10\% normalisation difference

$\rightarrow$ Within normalisation uncertainties of each measurement

- Comparison sensitive to systematics effect 


\section{experimental support of the Collins factorisation}

Look at the ratio of the diffractive to inclusive cross section

Observation: $Q^{2}$ dependence approximately similar for diff and incl...

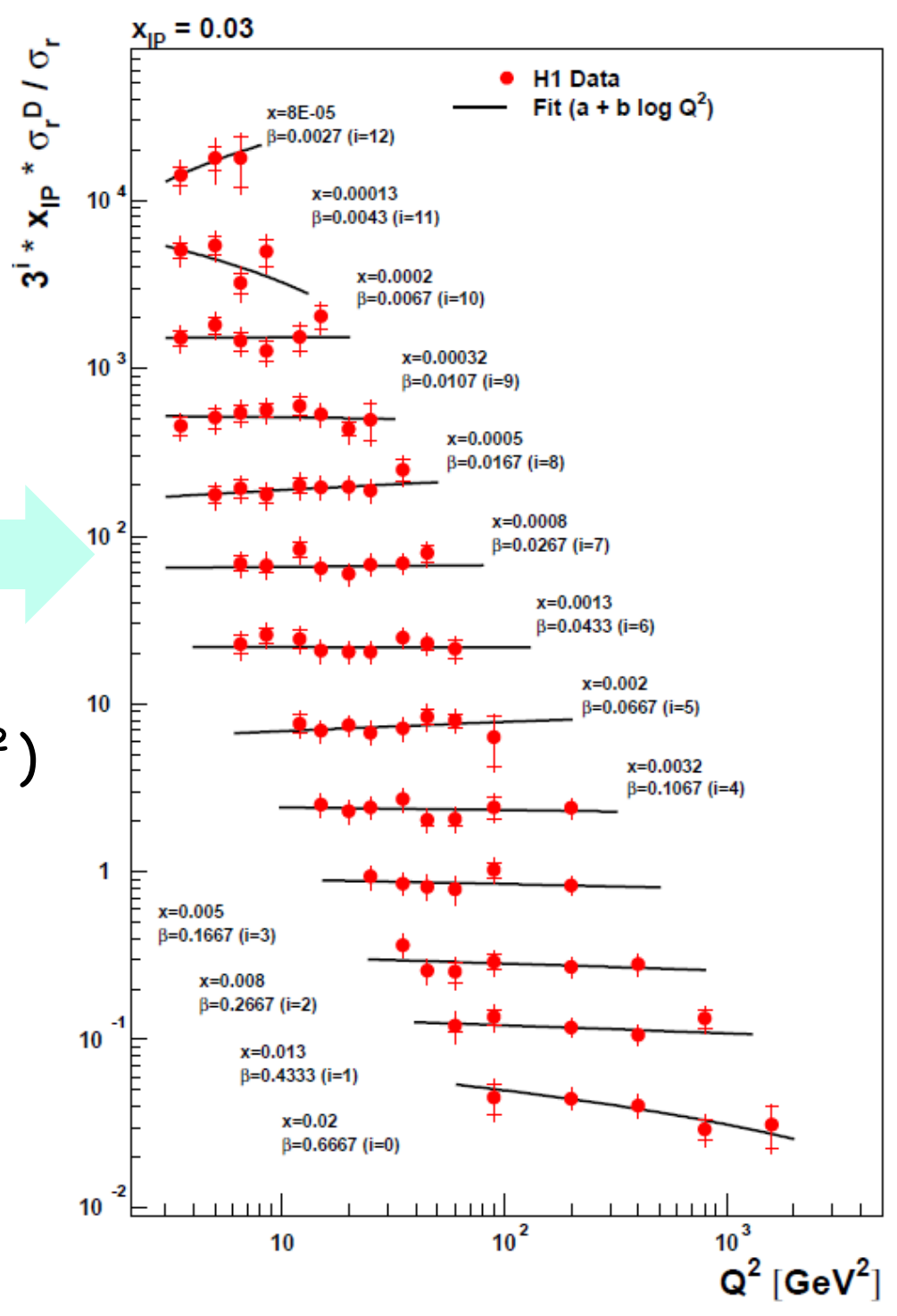

Support the fact that evolution equations $\left(Q^{2}\right)$ can be applied for diff...

(// standard inclusive F2) 


\section{QCD and diffraction (b)}

\section{'so-called' Regge factorisation (hypothesis) [Ingelman-Schlein]}

(b)

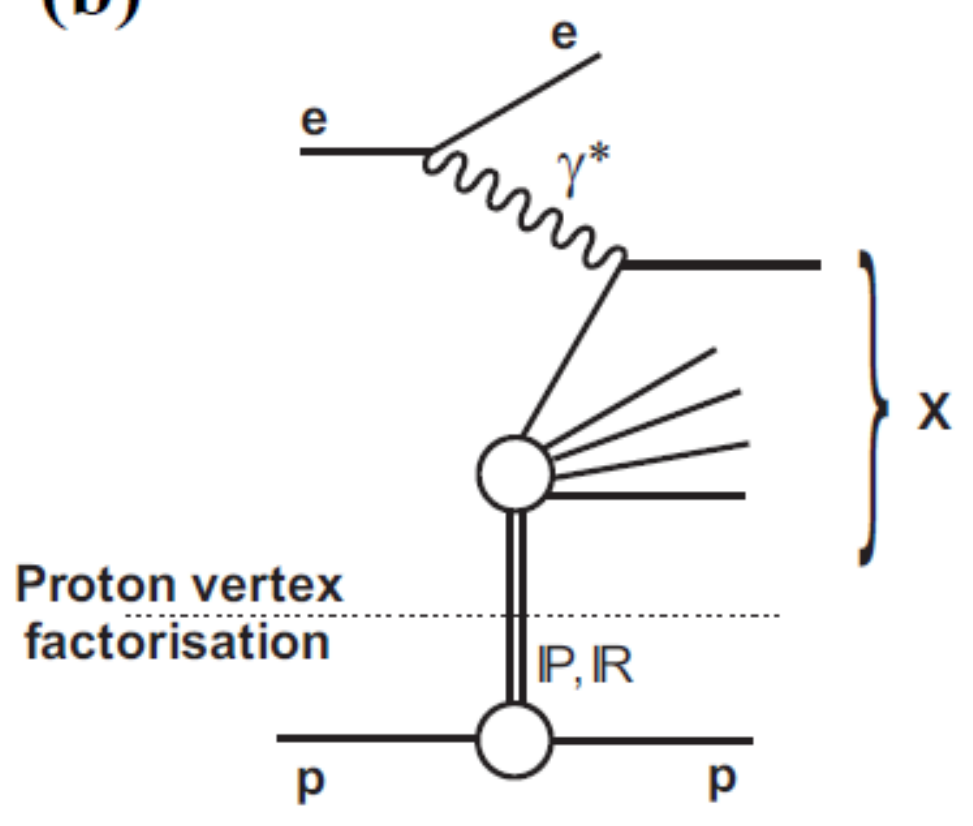

Assume:

$a^{\mathrm{D}}\left(x_{\mathbb{P}}, z, Q^{2}\right)=f_{\mathbb{P}}\left(x_{\mathbb{P}}\right) a^{\mathbb{P}}\left(z, Q^{2}\right)$

with

$f_{\mathbb{P}}\left(x_{\mathbb{P}}\right)=\int_{t_{\text {cut }}}^{t_{\min }} \mathrm{d} t \mathrm{e}^{B_{\mathbb{P}} t} x_{\mathbb{P}}^{1-2 \alpha_{\mathbb{P}}(t)}$

Parameters of the Pomeron flux function also determined from data...

From data: $\alpha_{I P} \sim 1.11$ and $B \sim 6 \mathrm{GeV}^{-2}$ 


\section{$\alpha_{\text {IP }}$ and $t$-slope determinations}
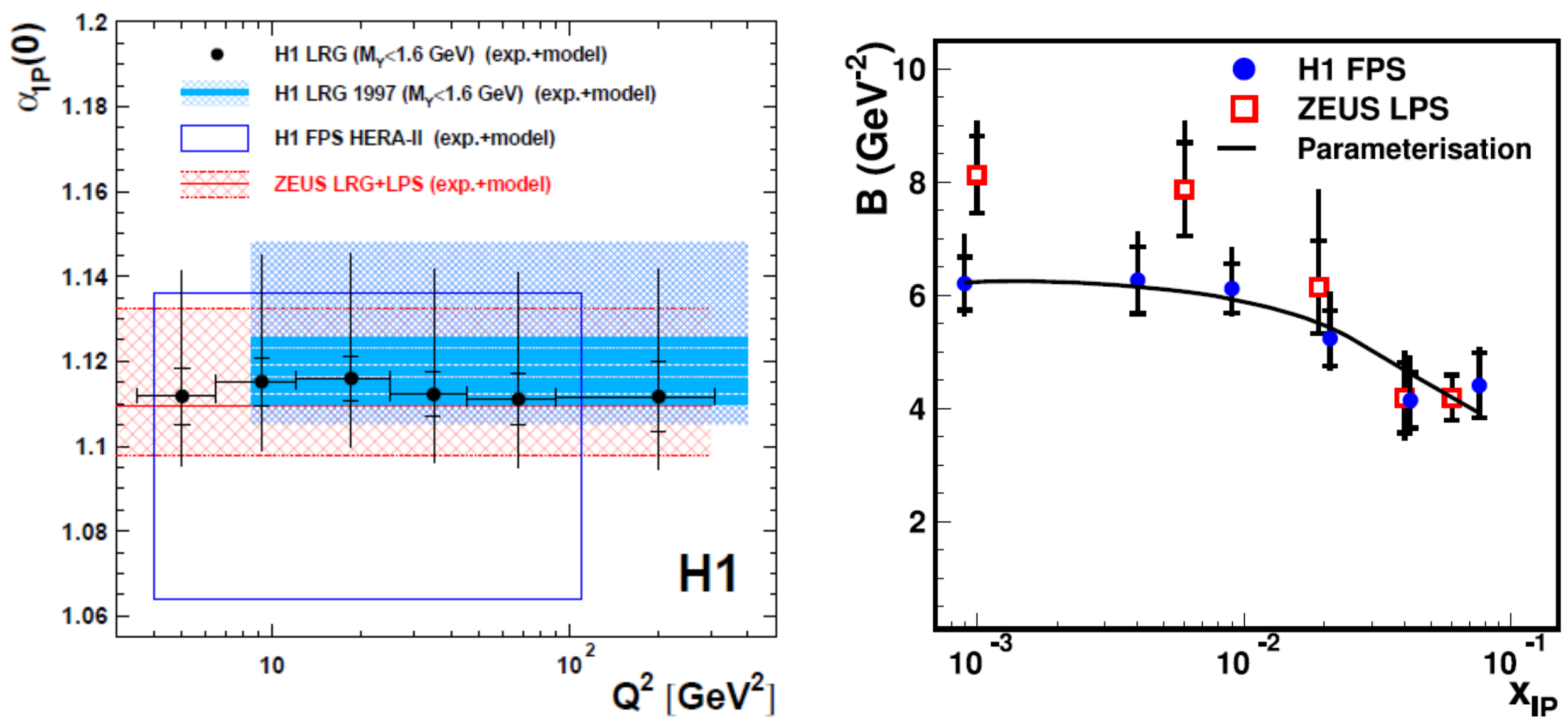


\section{Why the « Regge » factorisation is reasonable?}

$a^{\mathrm{D}}\left(x_{\mathbb{P}}, z, Q^{2}\right)=f_{\mathbb{P}}\left(x_{\mathbb{P}}\right) a^{\mathbb{P}}\left(z, Q^{2}\right)$

This means that if we divide $F_{2}{ }^{D}$ by $f_{I P}\left(x_{I P}\right)$ the dependence in $\left(z=\beta, Q^{2}\right)$ must be the same for all $x_{I P}$ values (small $\left.x_{I P}<10^{-2}\right) \ldots$

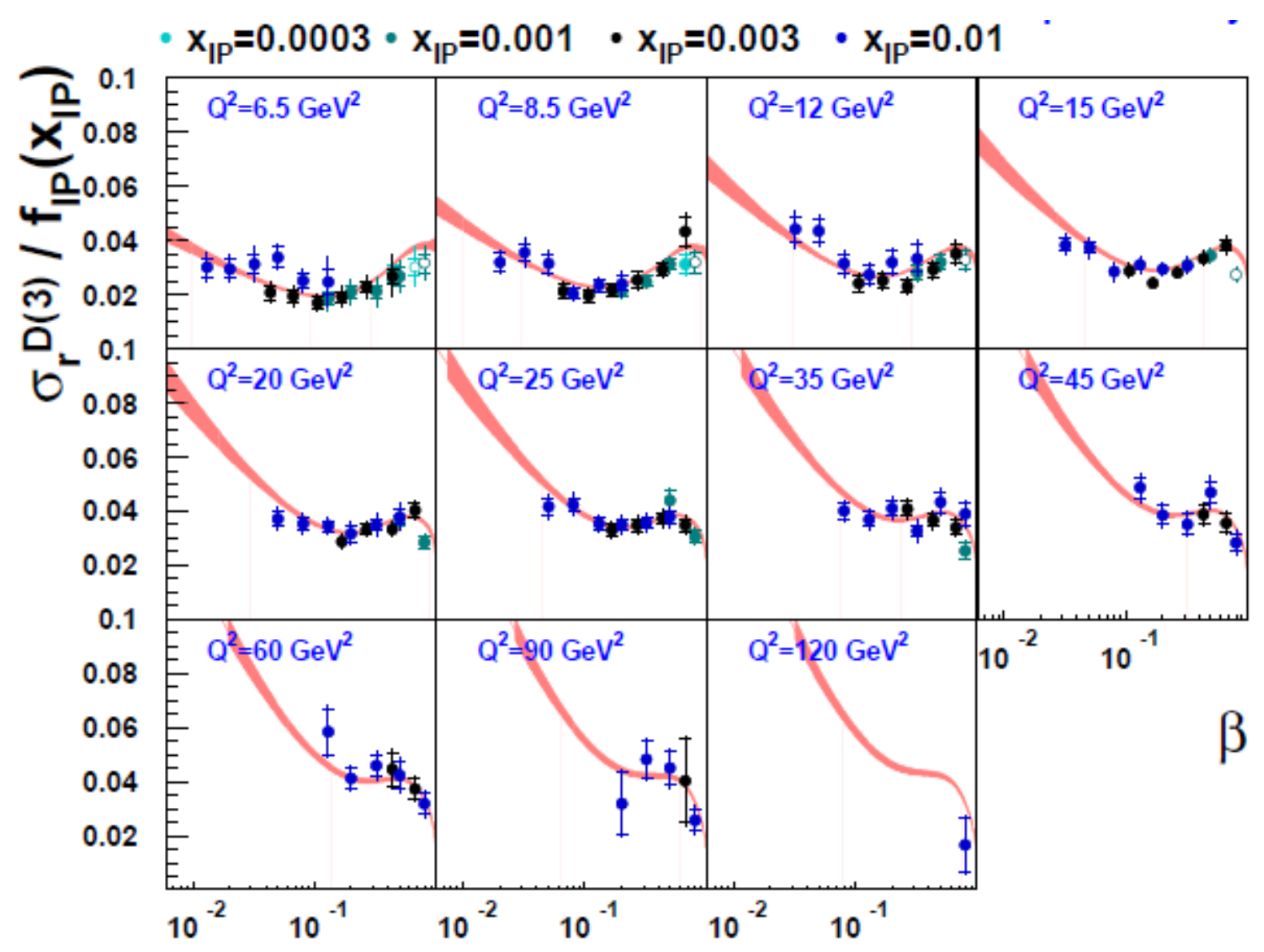




\section{Large $x_{I P}$ and sub-leading exchange}

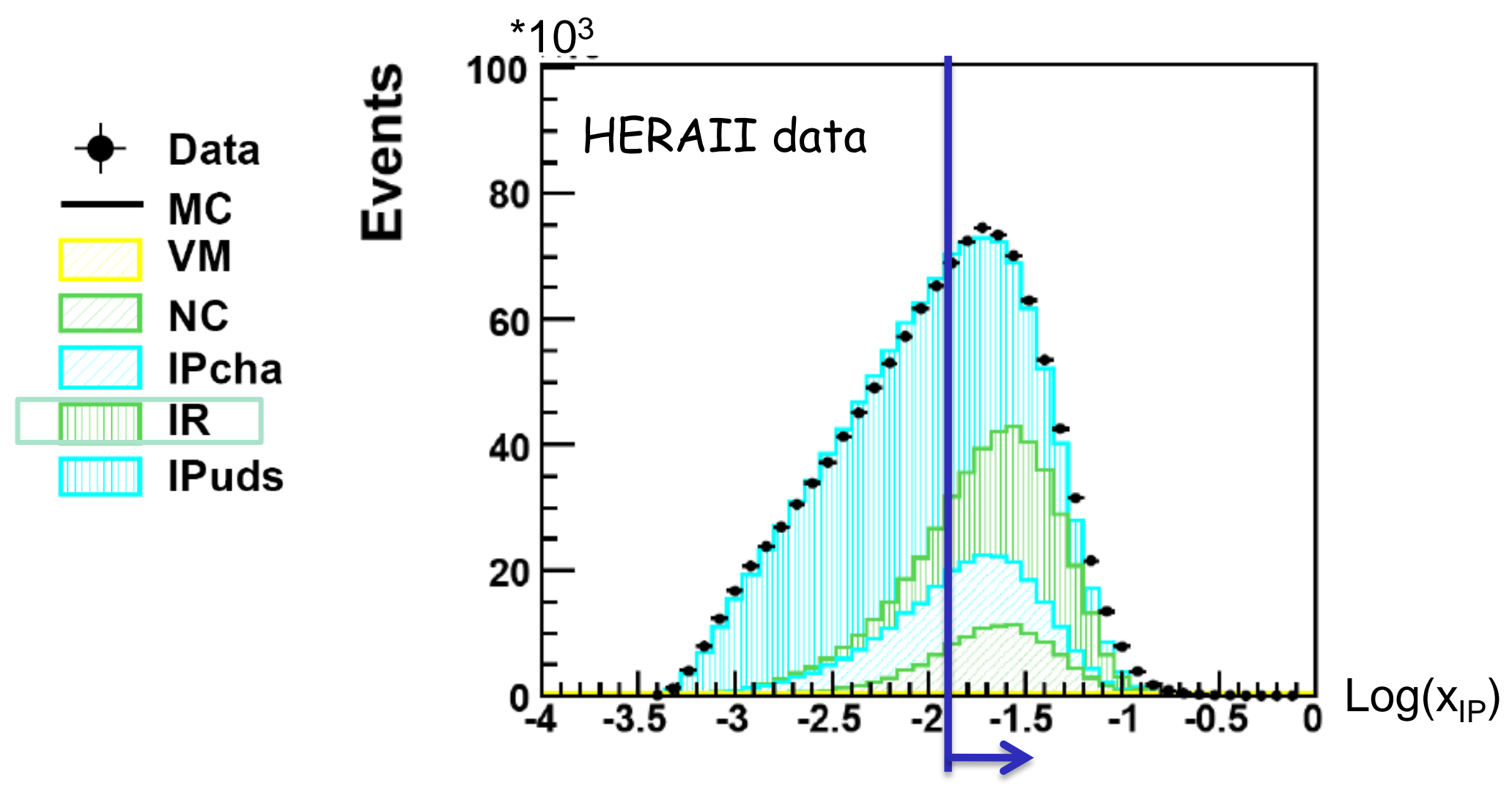

$x_{I P}>0.01 \Rightarrow$ contribution of Reggeons (IR) starts increasing (sub-leading exchange w.r.t. IP)

This is an irreductible background...

These IR lie on the approximately degenerate trajectory $\alpha_{I R}(t) \simeq 0.55+0.9 t$

...carry the quantum numbers of the $\rho, \omega, a$ or $f$ meson

...it is assumed that these exchanges can be expressed as the product of a flux and a meson structure function 


\section{Diffractive PDFs}

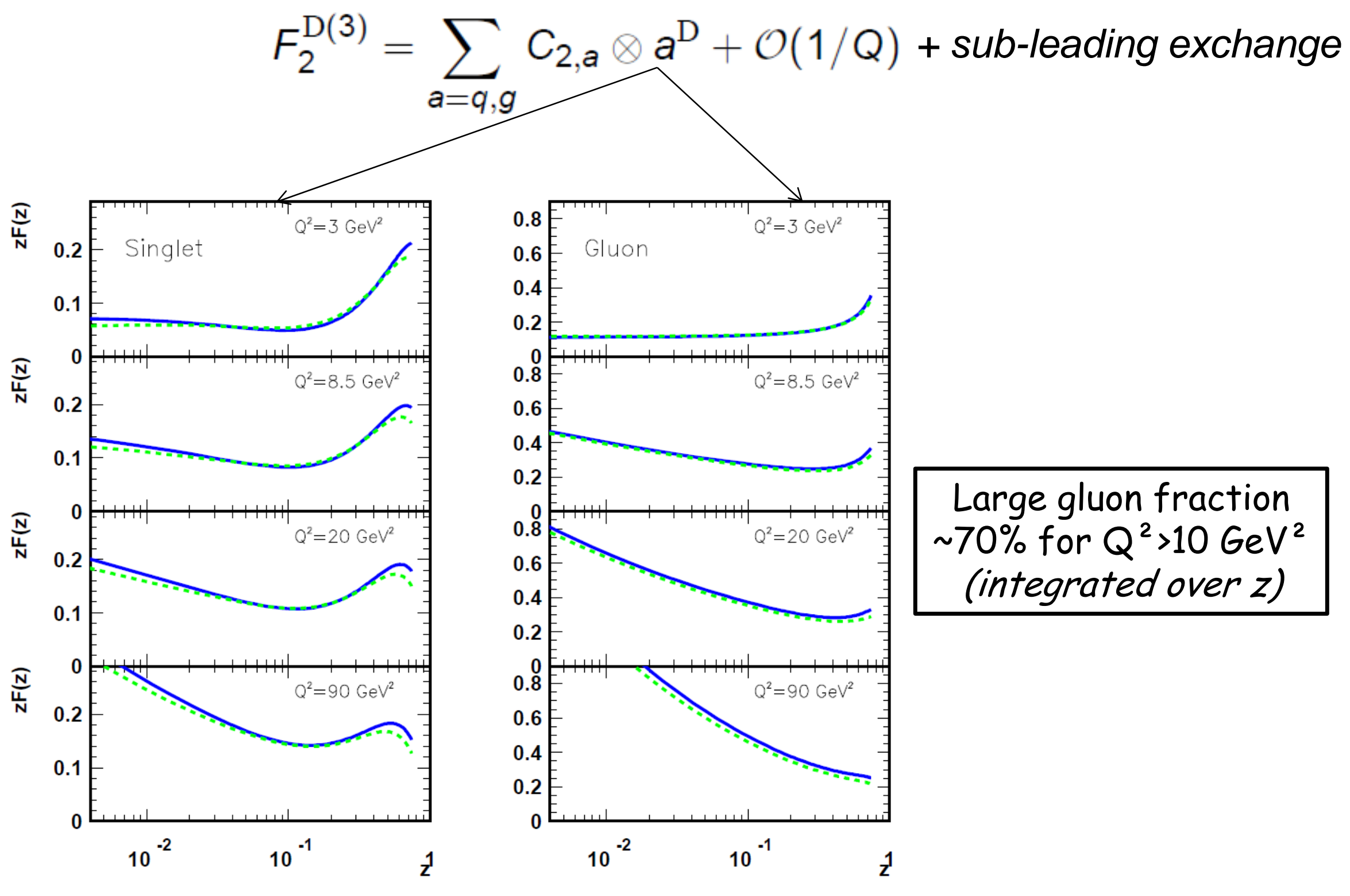




\section{Fit results $[\beta]$}

for $x_{\mid P}=0.01$
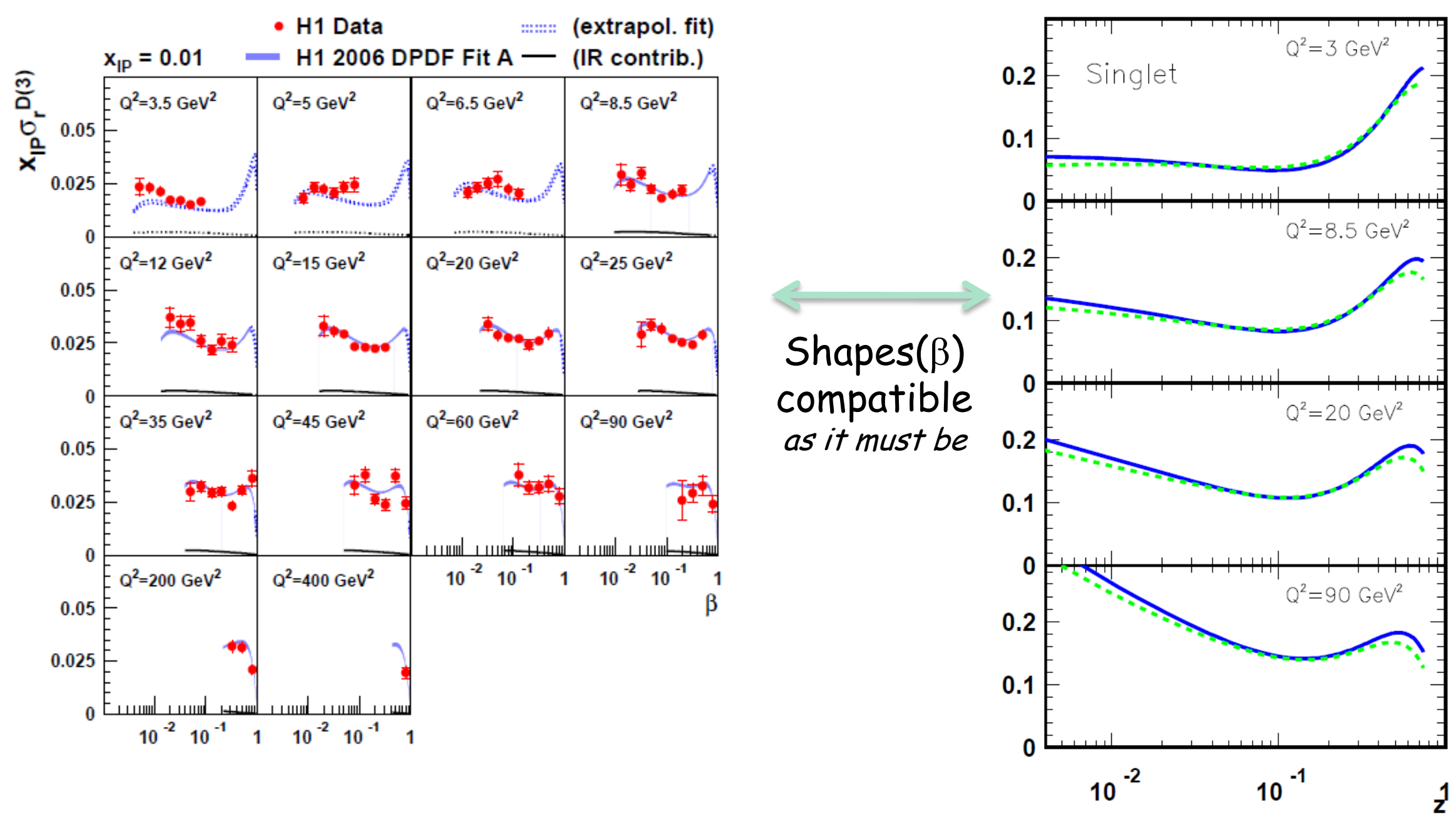


\section{Diffractive PDFs $(\mathrm{H} 1)$ from F2D only}
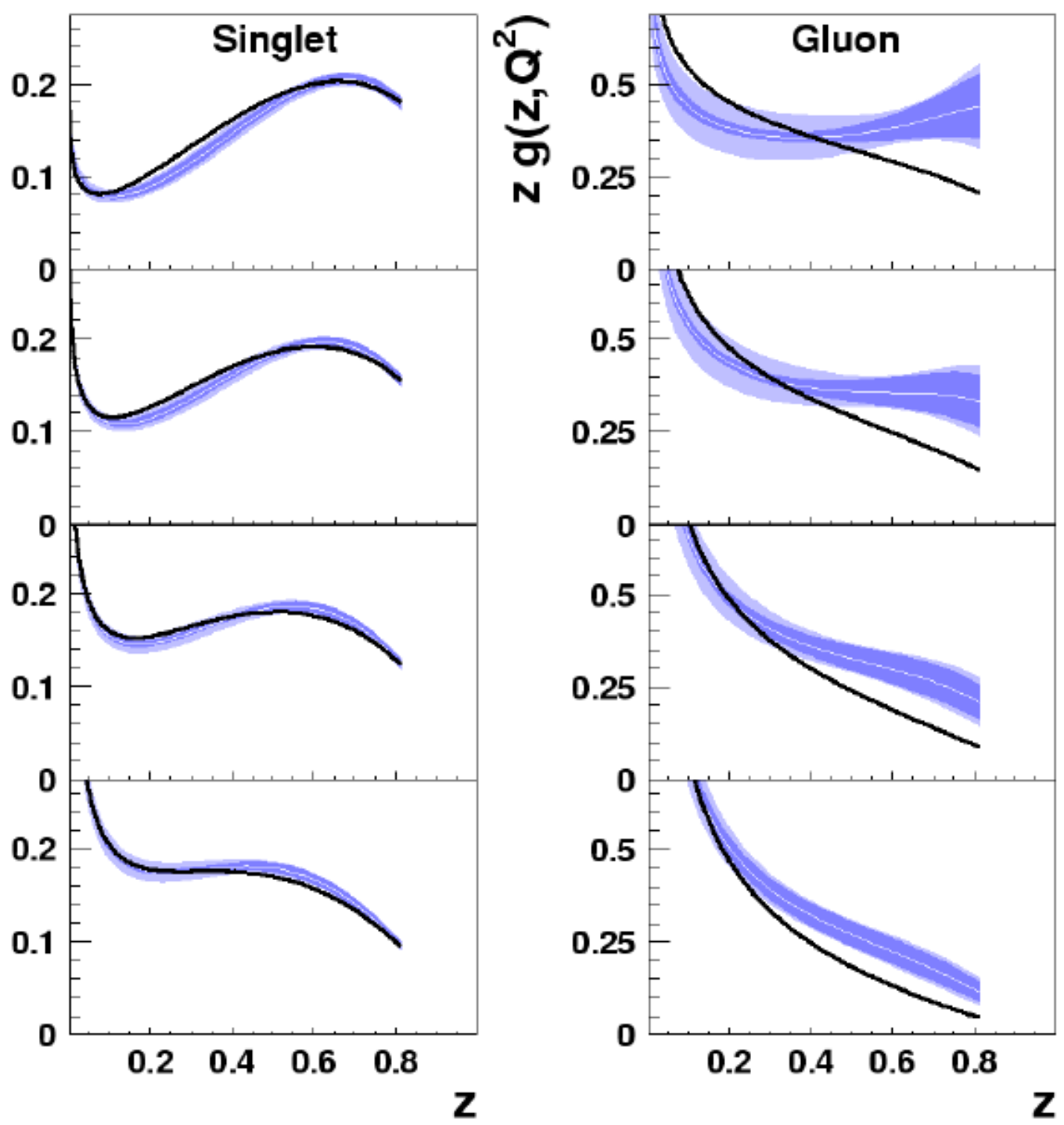

$Q^{2}$

$\left[\mathrm{GeV}^{2}\right.$

8.5

The ambiguity on the gluon distribution can be solved by including

20 Dijets cross sections 


\section{Diffractive dijets at HERA}

Clear sensitivity to the $z G$ hypothesis:

Fit A: large $z$ ' $z G$ ' solution of QCD fits

Fit $B$ : smaller ' $z G$ ' at large $z$

H1 data H1 2006 DPDF Fit A

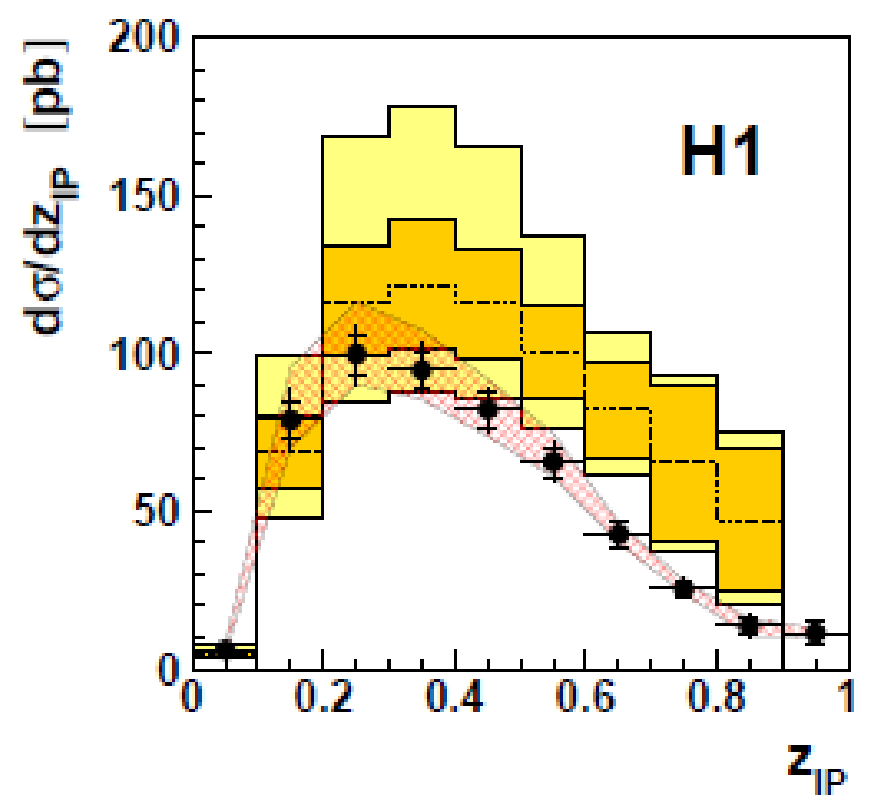

$\mathrm{e}\left(\mathrm{k}^{\prime}\right)$

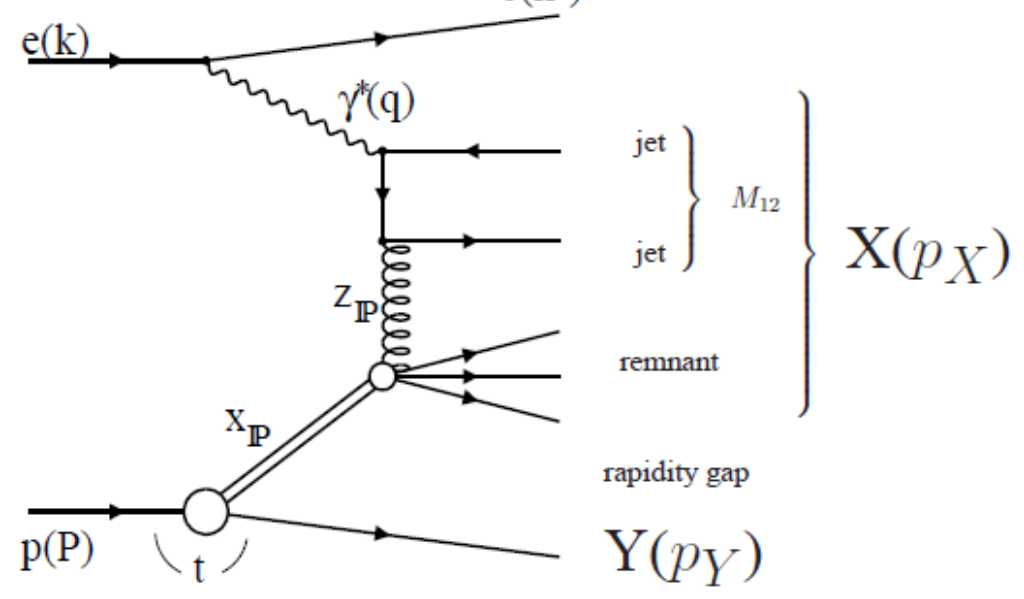

\section{H1 2006 DPDF Fit B}

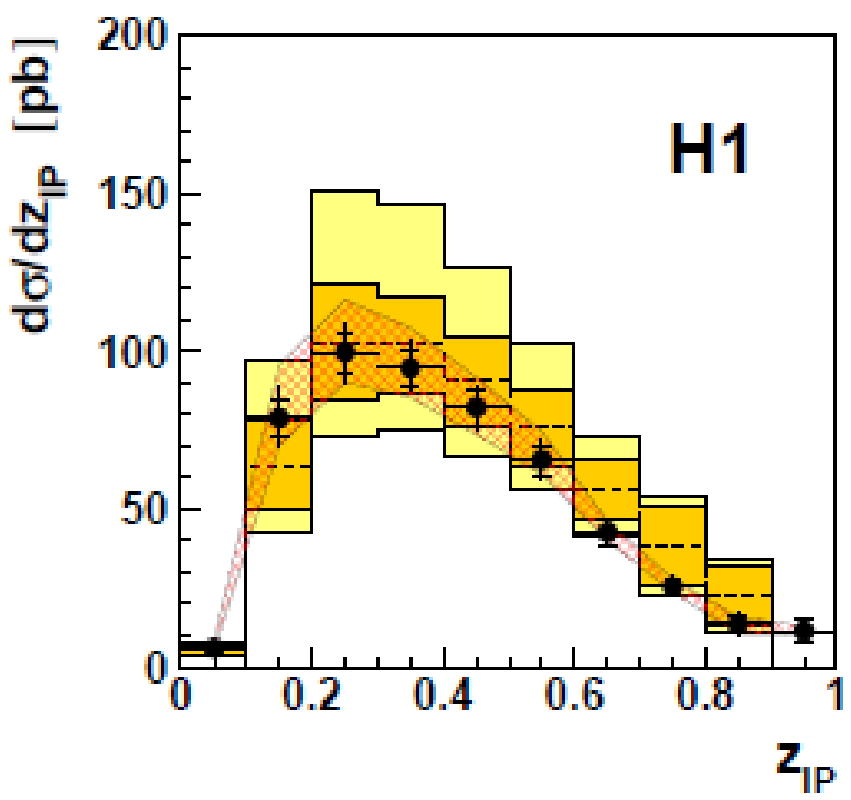

Use these data in the QCD fit in addition to F2D 


\section{Diffractive PDFs including jets}

- H1 2007 Jets DPDF

exp. uncertainty

exp. + theo. uncertainty

H1 2006 DPDF fit A

H1 2006 DPDF fit B
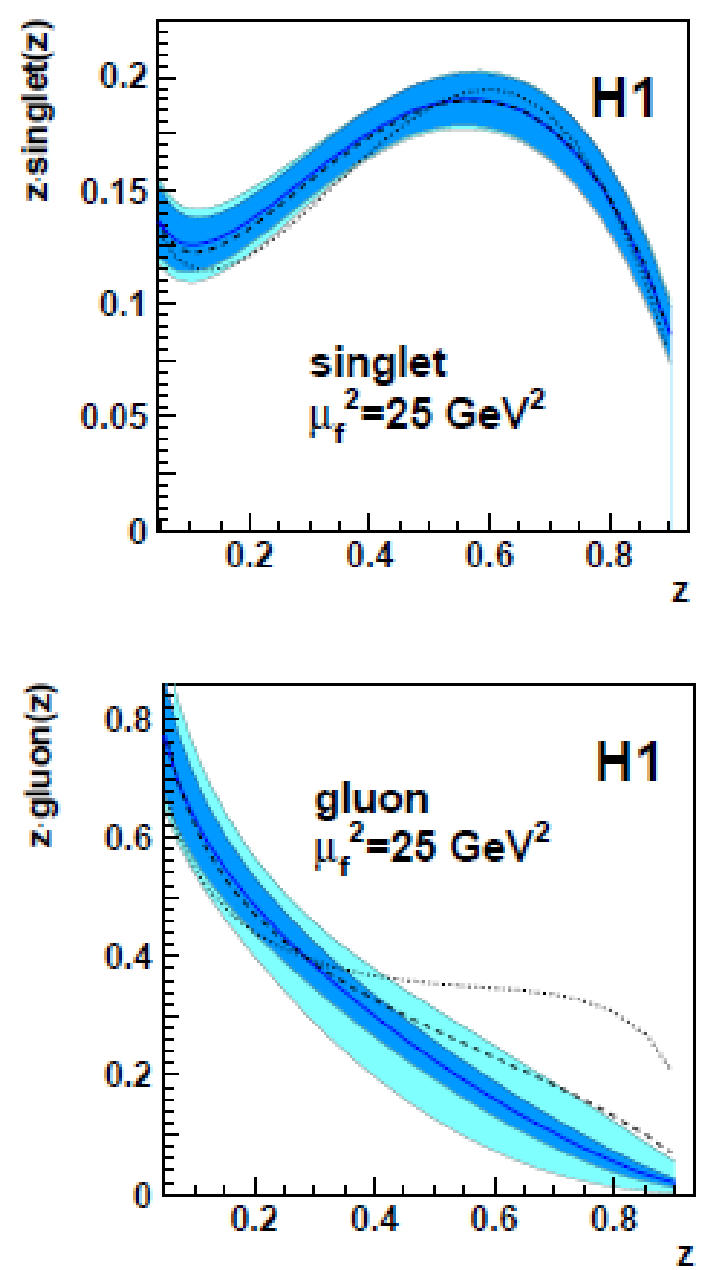

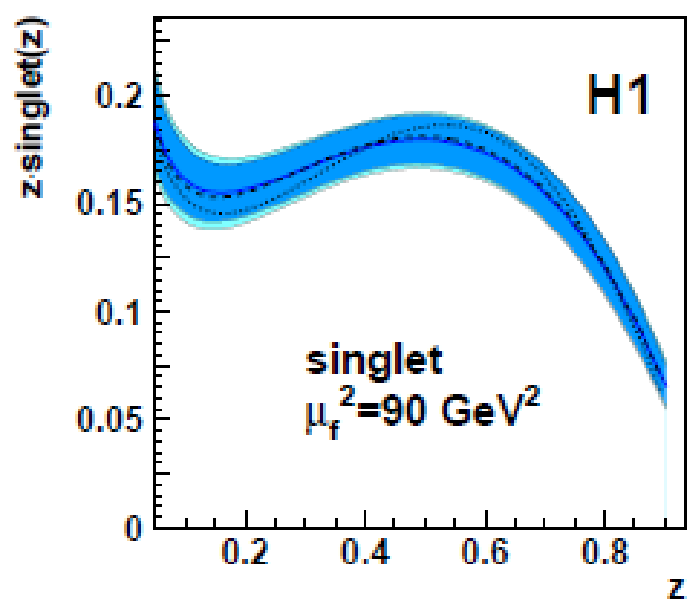

Now $z G$ is well constrained at large $z$

Compatibility with dPDFs including F2D only is shown on the figure...

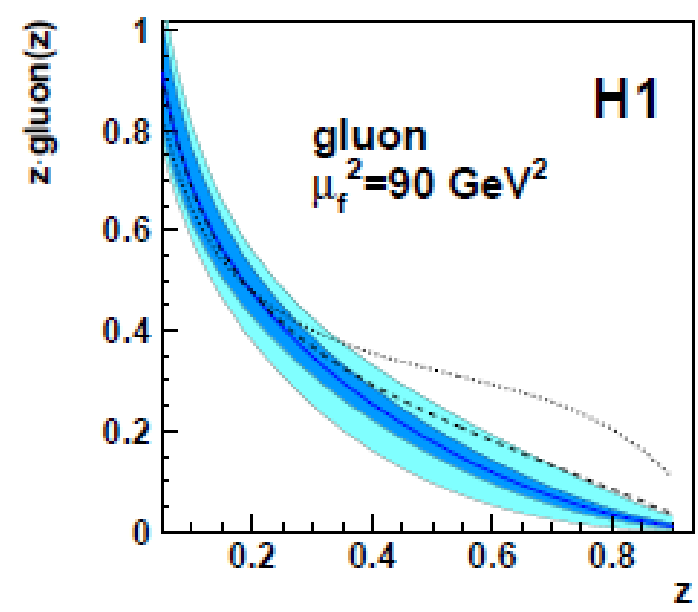




\section{Add-up: FLD measurements}

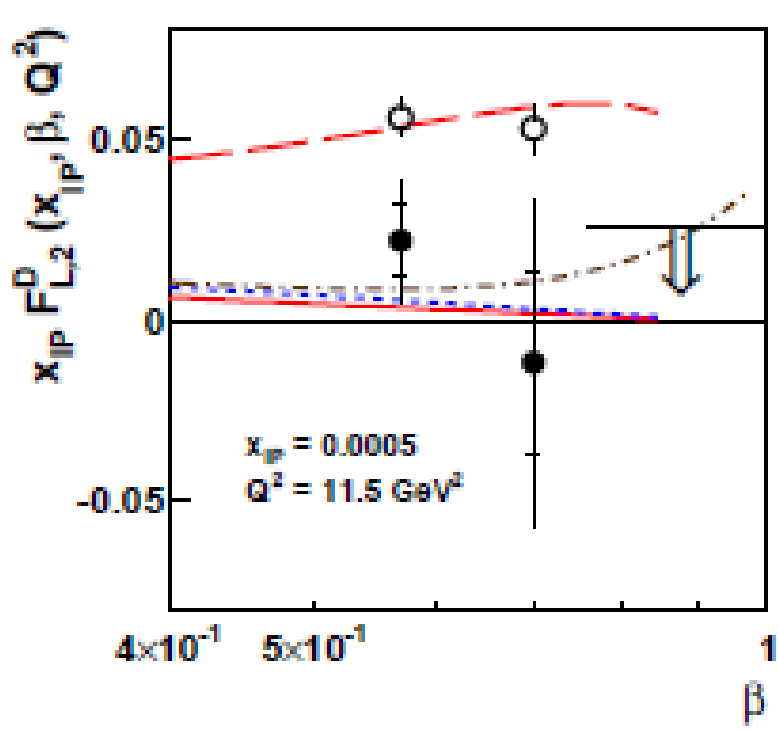

$$
\mathrm{x}_{\mathrm{IP}} \mathrm{F}_{\mathrm{L}}^{\mathrm{D}}
$$

- H1 data

$95 \% \mathrm{CL}$ upper limit

H1 2006 DPDF Fit B

H1 2006 DPDF Fit A

Golec-Biernat \& Luszczak

$\mathrm{X}_{\mathrm{IP}} \mathrm{F}_{2}^{\mathrm{D}}$

- H1 data

- H1 2006 DPDF Fit B
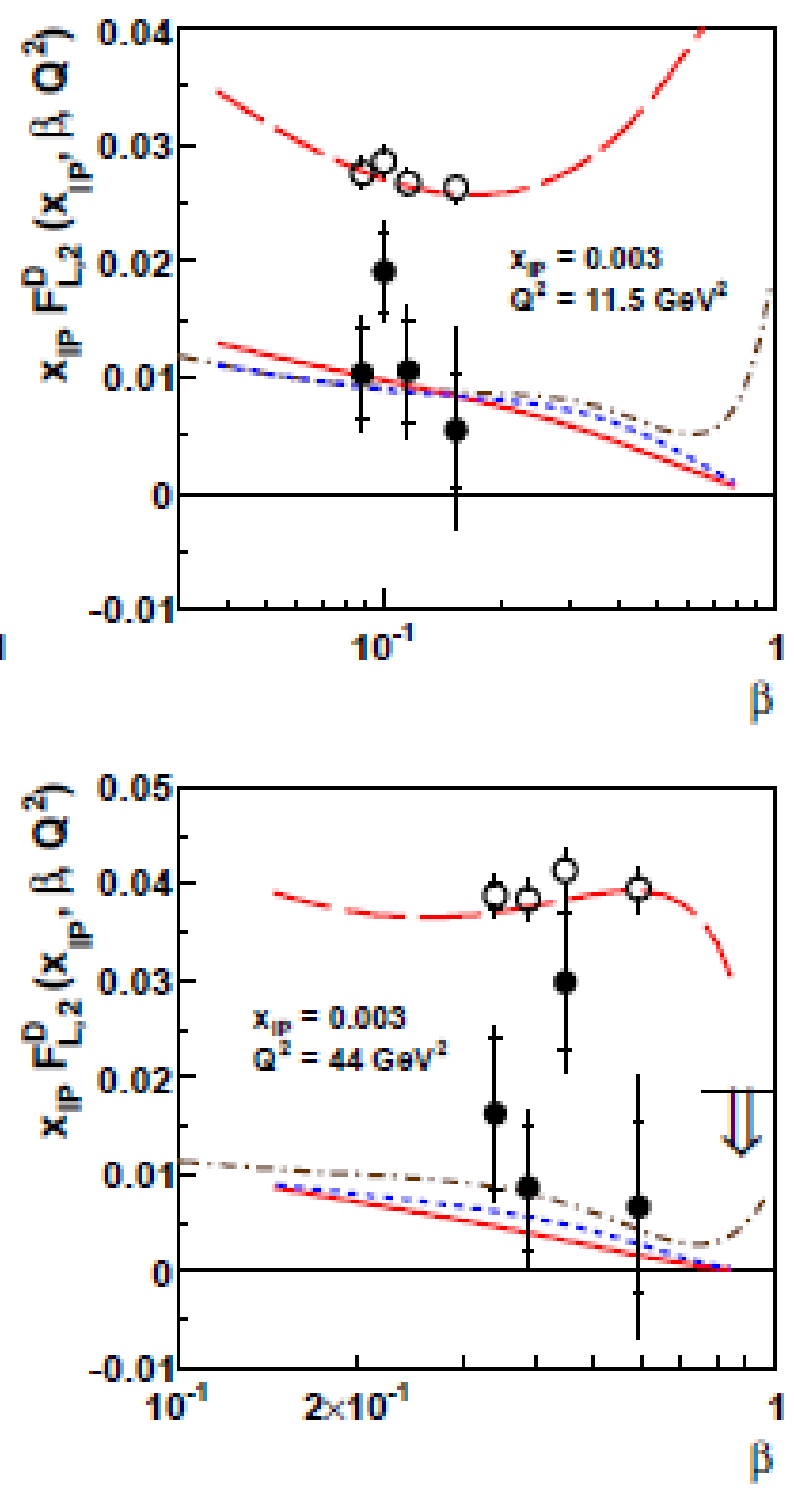

I can not describe the measurement, it would need a dedicated presentation.

In the context of this Talk $\Rightarrow$ we can check the size of FLD versus F2D and the good description by the diffractive PDFs (thus $z G$ )... 


\section{Conclusion -1-}

19 years after first HERA diffractive events ...

$H 1$ released its final LRG cross section measurement

$\rightarrow$ A precision measurement

$\rightarrow$ Reduced statistical and systematic errors

[H1 Coll. arXiv: 1203.4495]

- Amount of proton dissociation: $20 \%$

- New constraints for QCD models

- Data support the proton-vertex factorisation hypothesis

- Overall general agreement with ZEUS LRG data

Outlook: all HERA LRG data combination?

this is a nice project that will take time

Still a bunch of results to come from measurements using the VFPS 


\section{Conclusion -2-}

19 years after first HERA diffractive events ...

$H 1$ released its final LRG cross section measurement

$\rightarrow$ A precision measurement

$\rightarrow$ Reduced statistical and systematic errors

[H1 Coll. arXiv: 1203.4495]

Thanks to all $\mathrm{H} 1$ members

who worked during years

to make this measurement possible 